San Jose State University

SJSU ScholarWorks

Master's Projects

Master's Theses and Graduate Research

Summer 2021

\title{
Task Classification during Visual Search with Deep Learning Neural Networks and Machine Learning Methods
}

Siddartha Thentu

San Jose State University

Follow this and additional works at: https://scholarworks.sjsu.edu/etd_projects

Part of the Computer Sciences Commons

\section{Recommended Citation}

Thentu, Siddartha, "Task Classification during Visual Search with Deep Learning Neural Networks and Machine Learning Methods" (2021). Master's Projects. 1028.

DOI: https://doi.org/10.31979/etd.2vac-qhu5

https://scholarworks.sjsu.edu/etd_projects/1028

This Master's Project is brought to you for free and open access by the Master's Theses and Graduate Research at SJSU ScholarWorks. It has been accepted for inclusion in Master's Projects by an authorized administrator of SJSU ScholarWorks. For more information, please contact scholarworks@sjsu.edu. 
Task Classification during Visual Search with Deep Learning Neural Networks and Machine Learning Methods

\section{A Project}

Presented to

The Faculty of the Department of Computer Science

San José State University

In Partial Fulfillment

Of the Requirements for the Degree

Master of Science

By Siddartha Thentu

April 2020 
Siddartha Thentu

ALL RIGHT RESERVED 
The Designated Thesis Committee is pending approval on the Thesis Titled Task Classification during Visual Search with Deep Learning Neural Networks and Machine Learning Methods

By

Siddartha Thentu

APPROVED FOR THE DEPARTMENT OF COMPUTER SCIENCE SAN JOSE STATE UNIVERSITY

May 2021

Dr. Nada Attar, Department of Computer Science

Dr. Robert Chun, Department of Computer Science

Dr. Fabio Di Troia, Department of Computer Science 


\begin{abstract}
Studies have shown the possibility to classify user tasks from eye-movement data. We present a new way to determine the optimal model for different visual attention tasks using data that includes two types of visual search tasks, a visual exploration task, a blank screen task, and a task where a user needs to fixate at the center of any scene. We used deep learning and SVM models on RGB images generated from fixation scan paths from these tasks. We also used AdaBoost on filtered eye movement data as a baseline. Our study shows that deep learning gives the best accuracy for classifying between visual search tasks but misclassified between visual search and visual exploration tasks. Machine learningbased methods performed with high accuracy classifying tasks that involve minimal visual attention. Our study gives insight on the best model to choose by type of visual task using eye movement data.
\end{abstract}

Keywords - Human-centered computing, Visualization, Computing methodologies, Machine learning algorithms 


\section{TABLE OF CONTENTS}

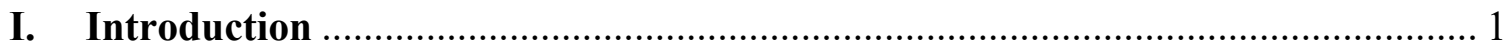

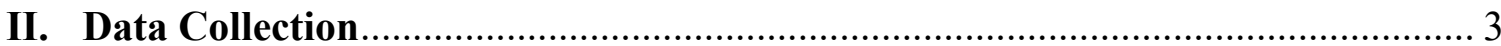

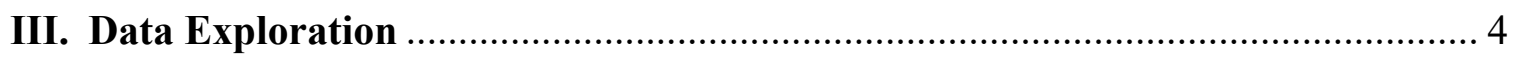

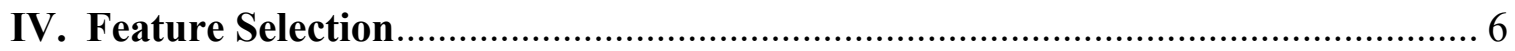

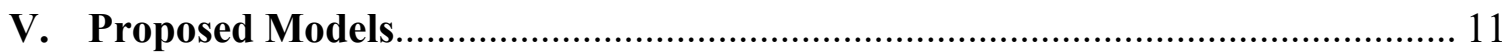
A. AdaBoost
B. $S V M$
C. $V G G-16$

VI. Experimental Methods
A. AdaBoost
B. $S V M$
C. $V G G-16$

VII. Conclusion and Future Work

References. 


\section{LIST OF FIGURES}

Figure 1: A sample of dataset used in the three models ............................................... 5

Figure 2: A subset of the Microsaccades data generated from the raw data..................... 6

Figure 3: Distributions of Pupil dilations................................................................ 7

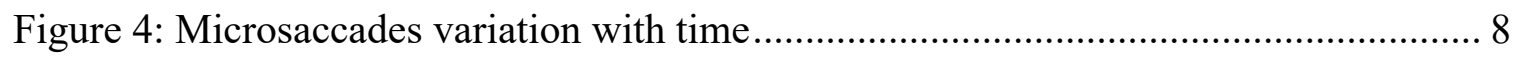

Figure 5: Average Microsaccade value across all four scenes .................................... 8

Figure 6: Corresponding RGB mappings of user gaze data .................................. 10

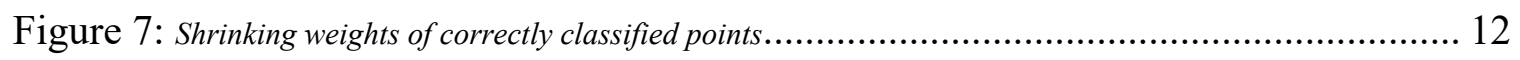

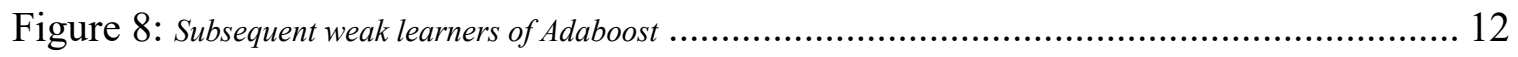

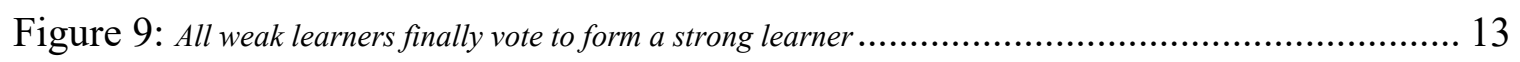

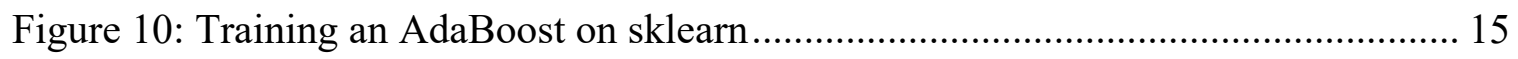

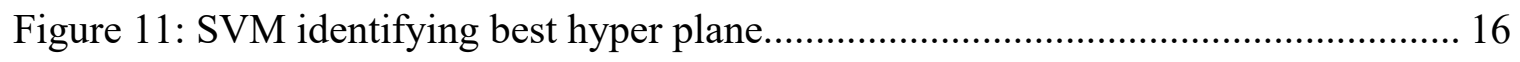

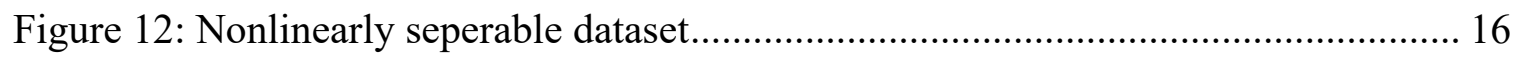

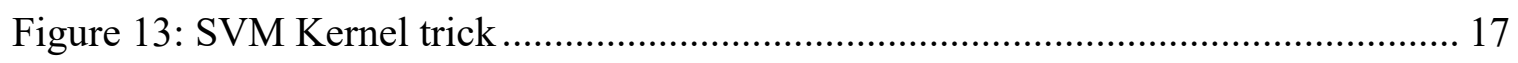

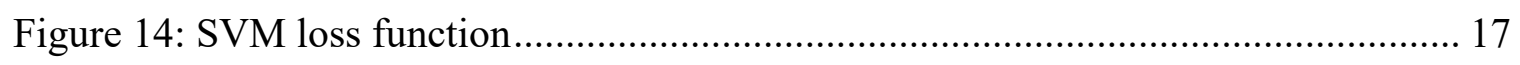

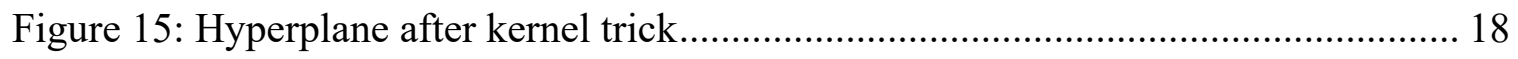

Figure 16: Implementing an SVM classifier with sklearn ........................................ 18

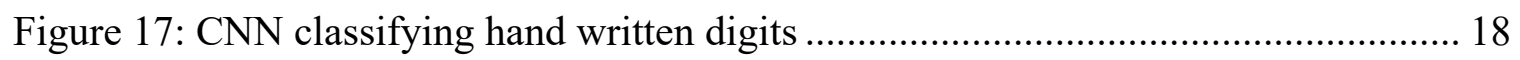

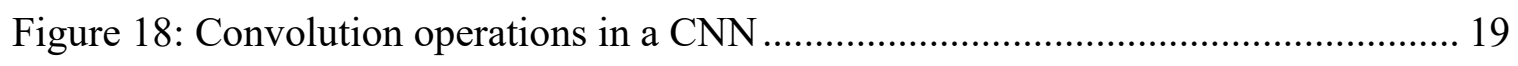

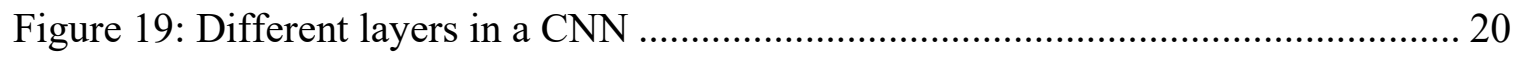

Figure 20: Kernel movement across source image ............................................... 21 
Figure 21: Convolution operations on a 3 dimensional image .................................... 22

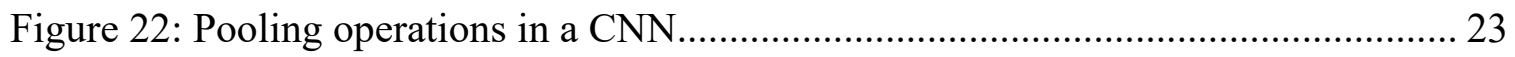

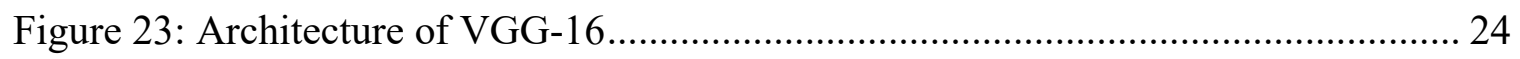

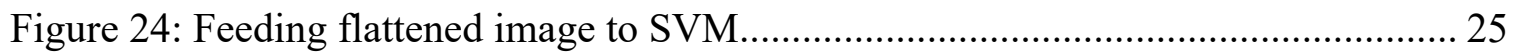

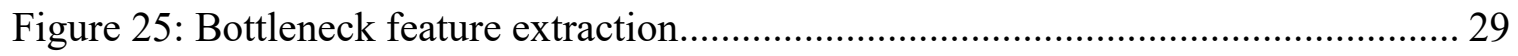

Figure 26: Feeding bottleneck features to SVM ..................................................... 30

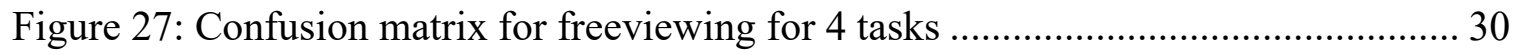

Figure 28: Confusion matrix for freeviewing for 3 tasks ....................................... 34

Figure 29: Confusion matrix for freeviewing for 2 tasks ...................................... 35

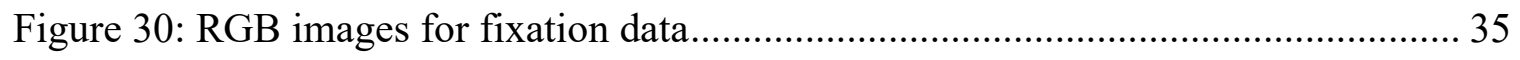




\section{LIST OF TABLES}

Table 1: Accuracy of models on microsaccade data................................................. 18

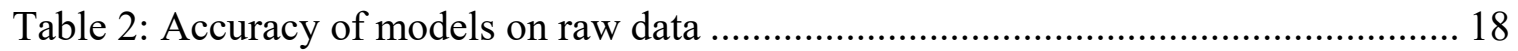

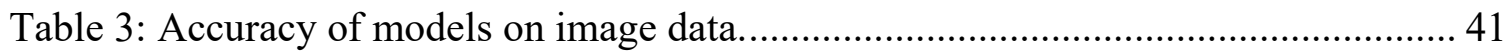




\section{INTRODUCTION}

One of the most common tasks that we arguably perform thousands of times every day is visual search. It has been one of the major paradigms for studying visual attention [A]. Researchers have used various visual search paradigms historically to gain insight into attentional selection in the visual system. In a typical visual search task, participants are asked to report if a visually distinctive target object is present among a set of distractors in a given scene. If the target and distractors have similar visual characteristics, observers must sequentially attend to search items in order to find the target or determine its absence. Many models of visual search have been proposed, aimed at explaining the role of visual attention (e.g., [5], [9], [12], [15]).

Yarbus presented qualitative data showing that eye movement patterns were dramatically affected by an observer's task in visual tasks. He suggested that complex mental states could be inferred from scan paths [1]. Several studies provided evidence that supports Yarbus's claim that eye movement during visual attention tasks can be used to predict user behavior [2], [11], [16], [20]. Another study by Greene et al. found that Yarbus's findings are arguable, and while it is possible for an observer's mental state to be decoded from some eye movement features, static scan paths alone are not sufficient to classify a visual attention task or to infer other complex mental states of the users [13]. In their study, they computed seven measures from the eye movement scan paths and fed them into a linear discriminant classifier. They failed to find any support for Yarbus's claim in their study. The eye movement patterns can differ across tasks, but not across images. This could account for the striking difference between Yarbus's result and Greene's. 
Several studies in addition to Yarbus and Green et al. have investigated deep learning models and other classification methods in visual attention tasks [3], [21], [23], [24], fixation pattern to predict scene category [18], or gaze fixations during visual search tasks [22]. The most recent study by Kumar et al. achieved $95.4 \%$ on task classification by eliminated highly correlated features before training an SVM and Ada Boosting classifier to predict the tasks from filtered eye movements data [6]. However, their results can also be argued. In their method, they used five correlated variables form the eye fixations, pupil size, and a label to classify the data based on the task. We think that this approach can be arbitrary as they only classified one task condition where subjects were instructed to only look at a fixation mark at the center of the scene. Therefore, classifying every single row of the data with five variables into either of the categories might not predict the visual search tasks [16]. A small set of eye movement variables or a singular example in a scan path could indicate the cognitive level at some state of the task but not necessarily the scanning patterns for an observer examining stimuli. In our work, we used the same AdaBoost model that was used by Kumar et al. We also used the same dataset that was collected from an extensive study carried out by Otero-Millan et al. [19]. We tested our model on the free-viewing condition that Kumar et al. eliminated from their study, where subjects can move their eyes freely in the stimuli during the visual search and visual exploration tasks. Our method also differed from previous studies in that we use RGB images of the scan path instead of extracting features. This allows us to use deep learning models that are effective for image classification. We used a ConvNet model and compared it with SVM and AdaBoost methods. 


\section{Data Collection}

Otero-Millan et al's dataset is compatible with our experimental design and meets the requirements of our goal of comparing between different classifiers on different visual tasks. The experiment included 2 different conditions and 4 different tasks. This would give a variation of visual tasks and variation in eye movement data. The experimental design was described in detail in [14][19]. The experiment has fixation conditions and freeviewing conditions. In the fixation conditions, subjects had to fixate a red cross on the center of the screen. In the free-viewing conditions, subjects were free to move their eyes over the visual scene. The four tasks are as follows: 1) Blank screen that showed a grey screen only, 2) Natural scene that has no target and subjects were instructed to explore the image, 3) Where's Waldo task, where the subjects performed a visual search task to find Waldo, 4) Picture puzzle condition, where subjects were required to find all the differences between two side-by-side, nearly identical images and indicate their locations at the end of the trial. Kumar et al used the fixation conditions only in their analysis, where the subject's task did not vary as they only need to look at the fixation mark at the center [6]. In the freeviewing conditions, the subject's task varied according to the visual scene presented. Each task had 15different visual scenes per the two conditions (except for the blank conditions). The total of the trials was 120 for each subject. The experiment was conducted over 3 sessions of 40 trials. 


\section{DATA EXPLORATION}

We included all the 8 subjects that are in the dataset. The data includes the pupil diameter and $\mathrm{x}, \mathrm{y}$ coordination of the fixation positions for the AdaBoost model. The data has the variables LP (left pupil), RP (right pupil), LX href (eye velocity for left eye on the x-coordinate), LY (eye velocity for left eye on the y-coordinate) href, LX Pix (pixel location for left eye on the x-coordinate) and LY Pix (pixel location for left eye on the ycoordinate). Each stimulus has the original image of $921 \times 630$ pixels on the screen. We filtered this data using the strategy carried by Kumar et al.in [6]. For the other two models, we generated images from the scan path of the eye fixations of both the left and the right eyes. We extracted the position of the eye based on LXpix, LYpix, RXpix, and RYpix. We calculated the eye position as of the X-coordinate $=$ ceil $($ LXpix + RXpix $) / 2$. Similarly, for the Y-coordinate $=$ ceil $($ LYpix + RYpix $) / 2$. This implies that at each timestamp, the user's eye was looking at a specific pixel that has two values. Then, these two values will be the row and column indices in the matrix for that scene. We inserted 1 in the matrix at the cell that corresponds to the row and column values. If the eye coordinate position calculated was beyond the edge of the image coordinates ( 621 by 930 ), the data will be simply ignored assuming that the user was looking outside the image scene. Finally, we would convert all the matrices into images. These images are the results of plotting locations according to the timestamp of the trials. Figure 1 shows a sample of each task and the correspondent RGB image of the scan path from the eye fixations for both conditions. The dataset consists of 120 images for each task and hence, 480 images are the total across all subjects. We rescaled those images to better work with the deep learning model known as ConvNet [5]. 
All the images were resized to $224 \times 224$. Most of the top performing ConvNet models like VGG16 or Inception V3 work on images of size 224 by 224 or 299 by 299 . These images with sizes require less computation overhead. Therefore, we choose images of size 224 by 224. activation function.
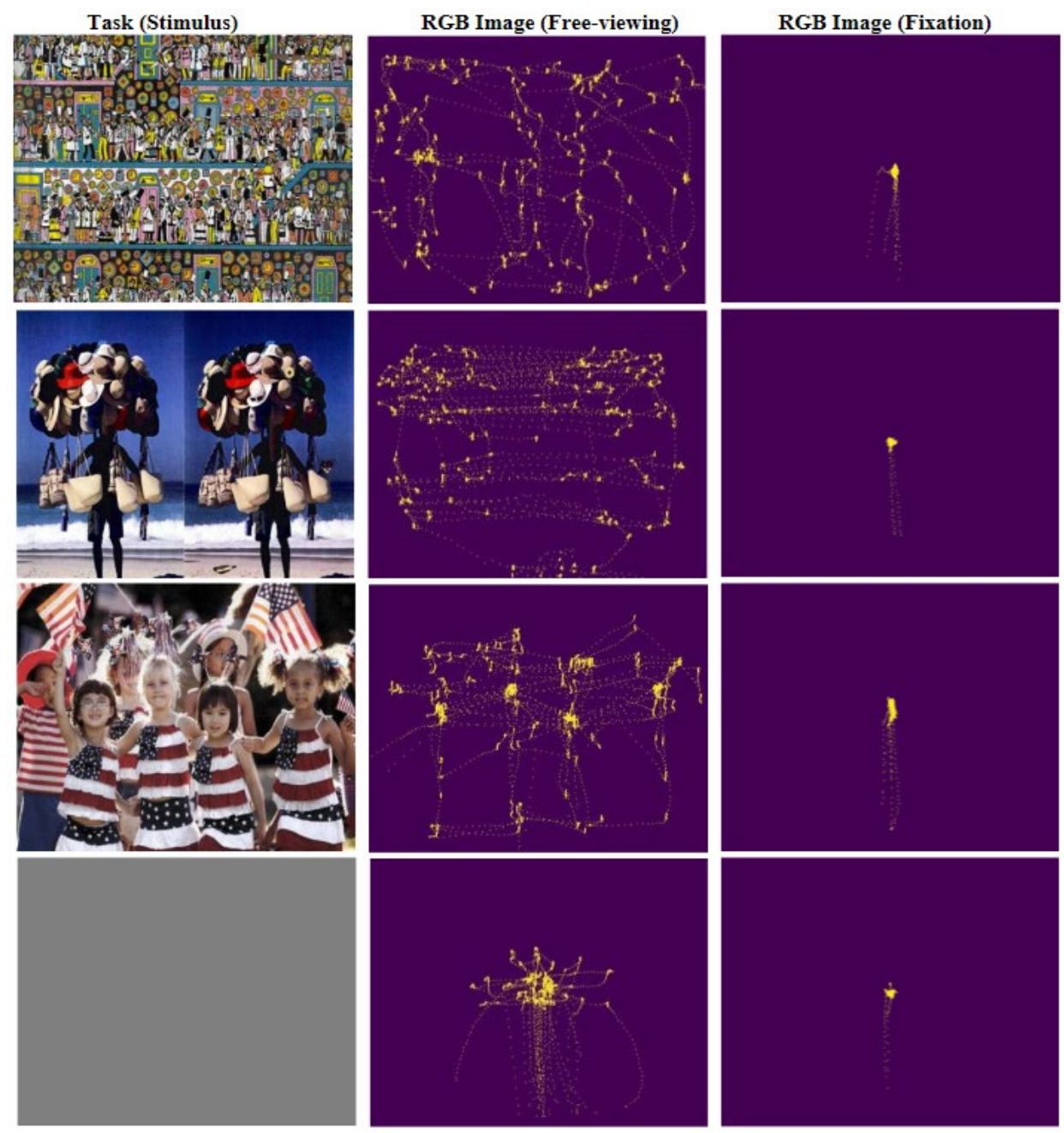

Figure 1: A sample of dataset used in the three models. Left: A sample of the actual task performed by the subjects. Center: An RGB image of the scan path of the fixation positions during the free viewing condition. Right: RGB image of the scan path of the fixation positions during the fixation condition. Rows: (A) Where's Waldo, (B) Picture Puzzle, (C) Natural Scene, (D) Blank Task (50\% gray) 


\section{Feature SELECTION}

\section{A. Microsaccades data}

The initial intention was to use the Microsaccades data and utilize machine learning algorithms to identify patterns in Microsaccades and pupil data to uniquely classify the data into either waldo, puzzle, natural or blank scenes. In other words, the machine learning algorithms should learn the Microsaccades and pupil patterns of all users (which will be the features) given the target labels for these patterns based on which scene the pattern belongs to. For example, a target label 0 would be assigned to all the data from Waldo, a target label 1 for puzzle, a target label 2 for natural and a target label 3 for Blank scene. The Microsaccades data has been extracted by running an algorithm on the raw data.

\begin{tabular}{|r|r|r|r|r|r|} 
Trial & \multicolumn{2}{|c|}{ BMicrosac LMicrosac } & RMicrosac LPupil & \multicolumn{2}{|c|}{ RPupil } \\
\hline 1 & 40 & 7 & 5 & 862 & 905 \\
\hline 1 & 1882 & 408 & 413 & 790 & 842 \\
\hline 1 & 266 & 77 & 114 & 229 & 279 \\
\hline 1 & 43 & 20 & 63 & 231 & 279 \\
\hline 1 & 44 & 23 & 63 & 231 & 307 \\
\hline 1 & 54 & 26 & 62 & 247 & 321 \\
\hline
\end{tabular}

Figure 2: A subset of the Microsaccades data generated from the raw data. From left to right the columns indicate binomial, left, right microsaccades and the pupil dilations of left and right eye

On comparing the pupil dilations across all four scenes of Waldo, Puzzle, Natural and Blank, we could not find significant differences that could strongly support pupil dilations as a contributing feature. Evidence shows that the distribution of pupil dilations were more or less the same across the four scenes. 


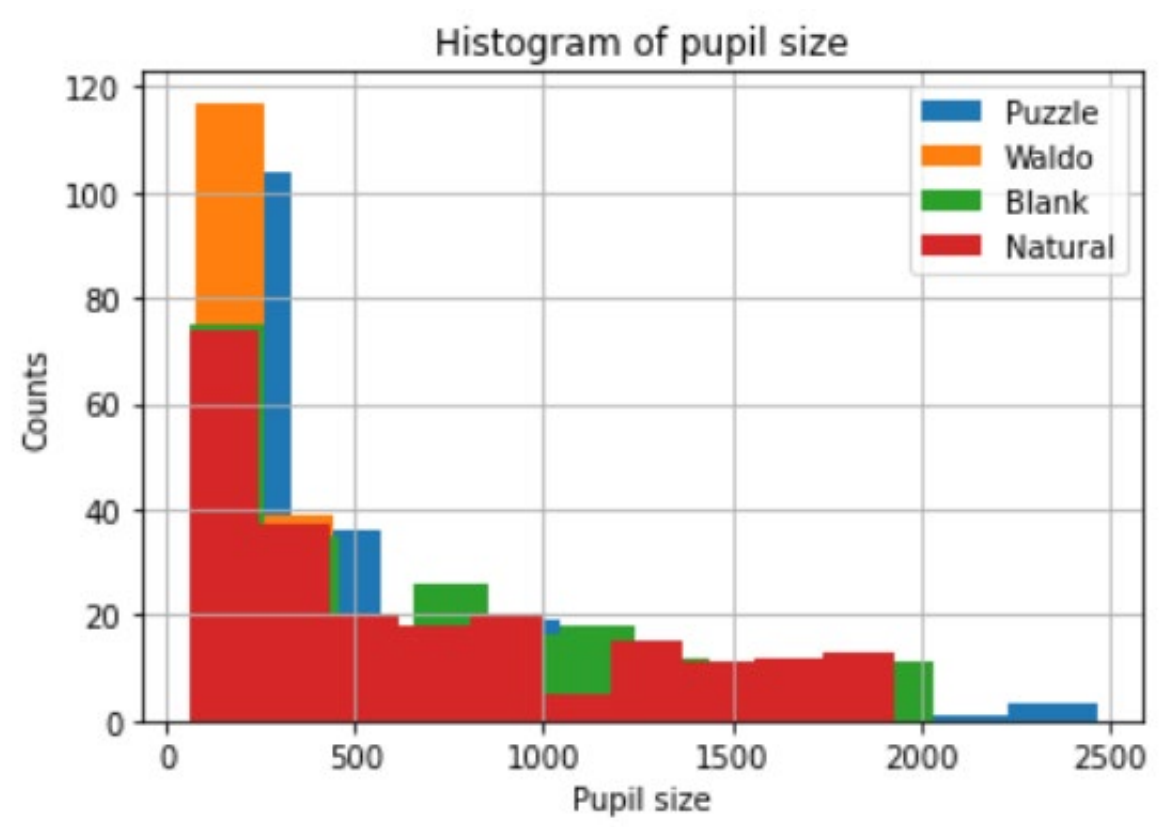

Figure 3: Distributions of pupil dilations across puzzle, waldo, natural and blank scenes

Figure 3 shows the similar distribution of pupil dilations across all four scenes. This evidence corroborates our idea that pupil dilations may not be a reliable feature in uniquely identifying the scenes. Features like these add noise to the data and make it hard for the model to identify patterns in the data. Furthermore, current medical evidence shows that pupil dilations are mostly affected by the lightning conditions but not by the visual challenges perceived by the subject. A similar pattern was found with the Microsaccades data across four scenes. Figure 4 shows that the Microsaccades variation with respect to time was almost similar across scenes like Puzzle, Waldo and Natural. The similarity in mean values of Microsaccades as shown in figure 5 also supports our claim that Microsaccades alone might not be a strong feature in scene classification. 


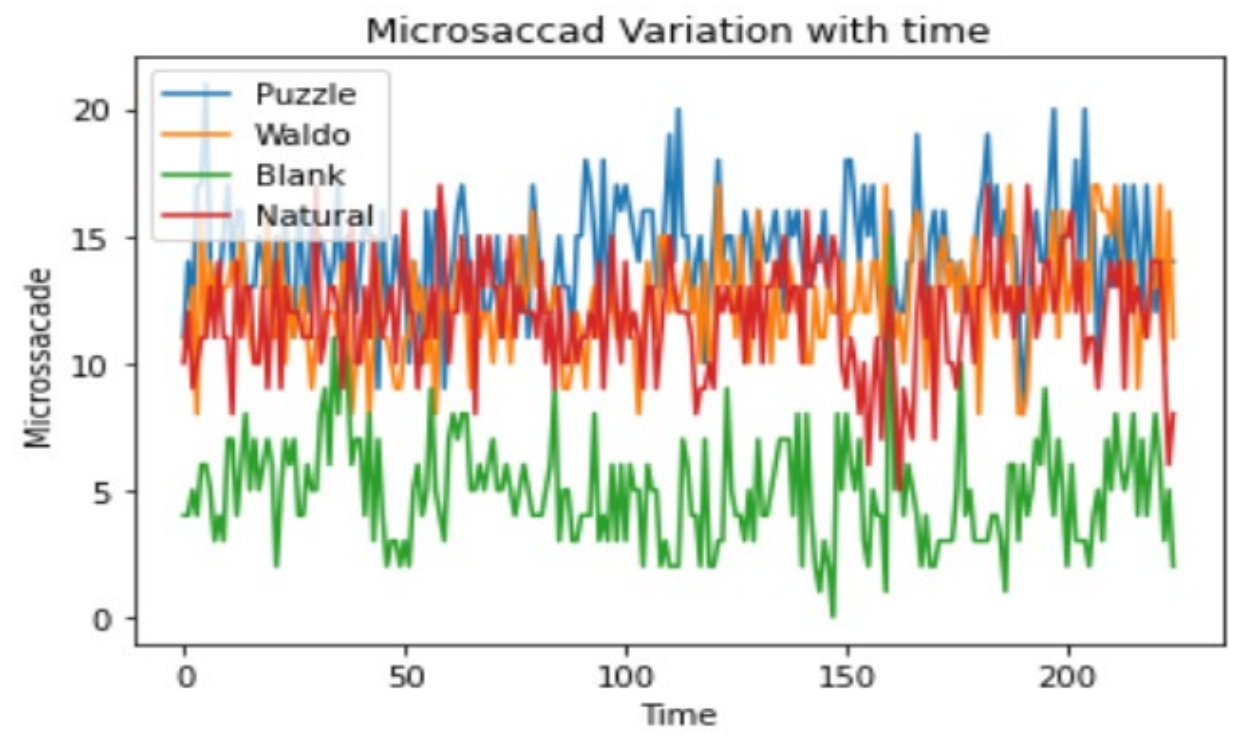

Figure 4: Microsaccades variation with time across four scenes

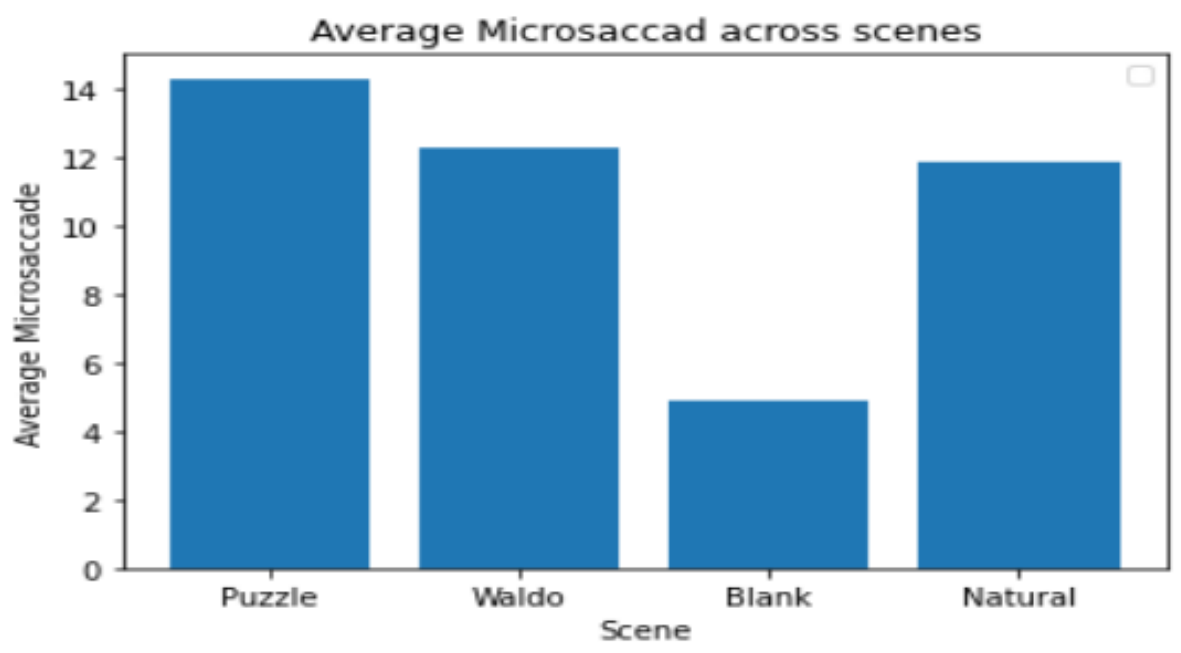

Figure 5: Average microsaccade value across all four scenes 
Table 1: Accuracy of models when run on the microsaccade data

\begin{tabular}{|c|c|}
\hline Method & Accuracy \\
\hline SVM & $40 \%$ \\
\hline Ada Boost & $50 \%$ \\
\hline
\end{tabular}

Table 1 shows that based on Microsaccades and Pupil data, machine learning algorithms performed poor in classifying the scenes.

\section{B. Raw data}

Although we employed the machine learning models on Microsaccades data generated from raw data, [4] achieved high results by just using the raw data. Since [4] experimented on fixation data, we tried to re-run the same models with the same configurations on freeviewing data. This method showed a boost in accuracy, but the results were not on the same level as fixation data.

Table 2: Accuracy of models when run on the raw data.

\begin{tabular}{|l|l|l|l|}
\hline Classes & Model & Fixation & FreeView \\
\hline 4 & AdaBoost & 95 & 84 \\
\hline 3 (excluding Natural) & AdaBoost & 97 & 88 \\
\hline
\end{tabular}

Since the raw data was a timeseries data of how a subject's gaze shifted across over a period, we strongly suggest [4]'s approach to treat a single time step as an individual experiment and employ machine learning models on the tabular data is wrong. We needed a novel approach to transfer the problem domain from classifying based on timeseries to image classification. 


\section{RGB Mapping}

The other approach we decided to take is to map the raw eye gaze data into RGB images. Utilizing this approach lets us make us of powerful image classification algorithms like SVM and Convolutional Neural Networks.
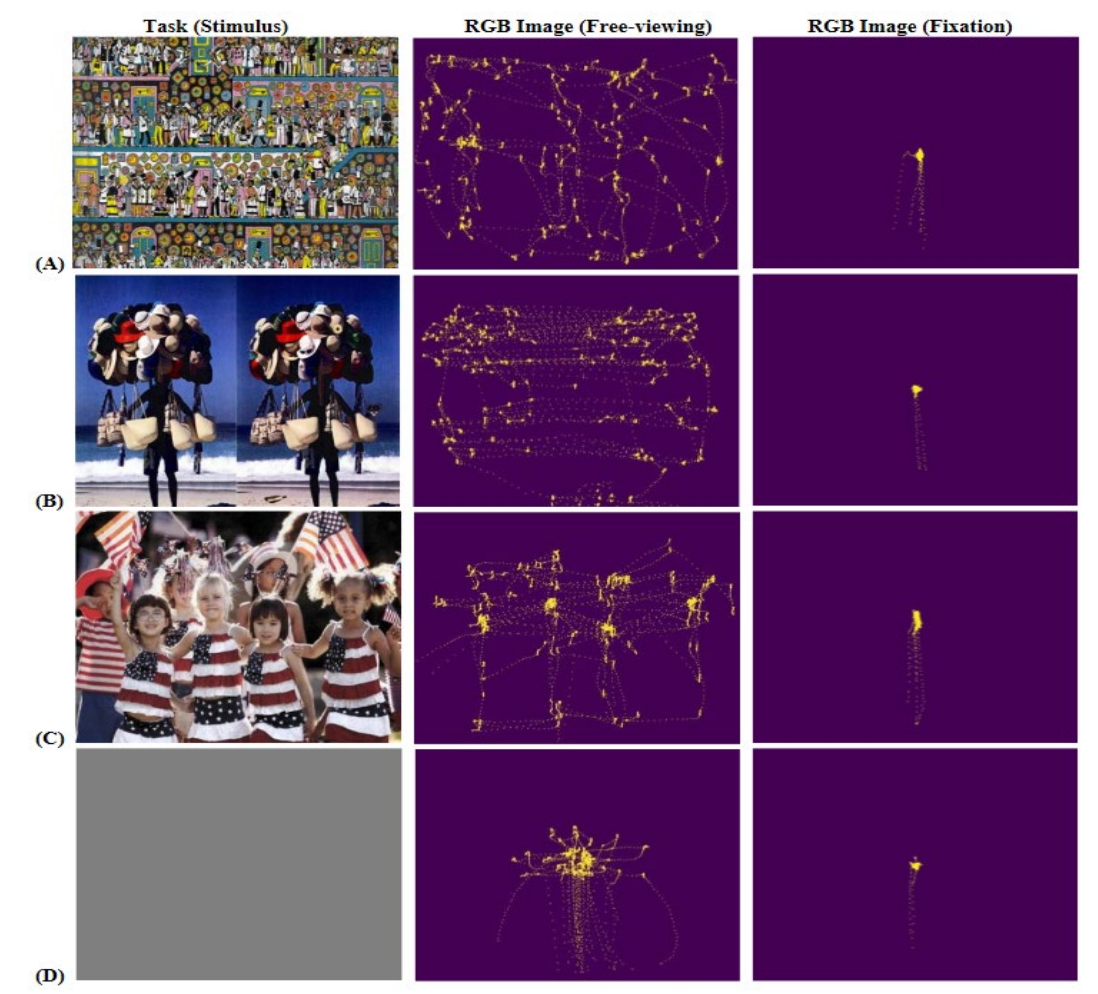

Figure 6: Corresponding RGB mappings of user gaze data for free viewing and fixation condition

Since the images generated for each scene were promisingly different, we were confident that power image classification algorithms like SVM's and CNN's will give better results. 


\section{Proposed Models}

Some of the popular algorithms used in our approach are AdaBoost, SVM and CNN's. In this section, we explore the fundamentals of the algorithms.

\section{A. AdaBoost}

Boosting is an ensemble learning model like bagging, except the classifiers are not picked at random. In Boosting algorithms, each classifier is picked in a way that focuses on the weaknesses of the previous classifiers. Each classifier is not necessarily strong, but the union of them is. One way this is done is by modifying the data by giving more weight to the misclassified points and less to the correctly classified ones and fit the next classifier to this modified data. Many ensemble methods started in order to prevent overfitting in decision trees, and for this reason decision trees tend to be more popular for this kind of approaches. In Boosting, the weak learners are picked in an intelligent way. Each weak learner is trained to focus on the misclassifications generated by the previous learner. In other words, each learner tries hard to correctly classify the points in which the previous classifiers have failed. This process starts by training an initial learner. Then the correctly classified data points are looked at. These correctly classified points are reduced by weighting them by a number smaller than 1 . Then the misclassified points are enlarged by weighting them by a number larger than 1 . 


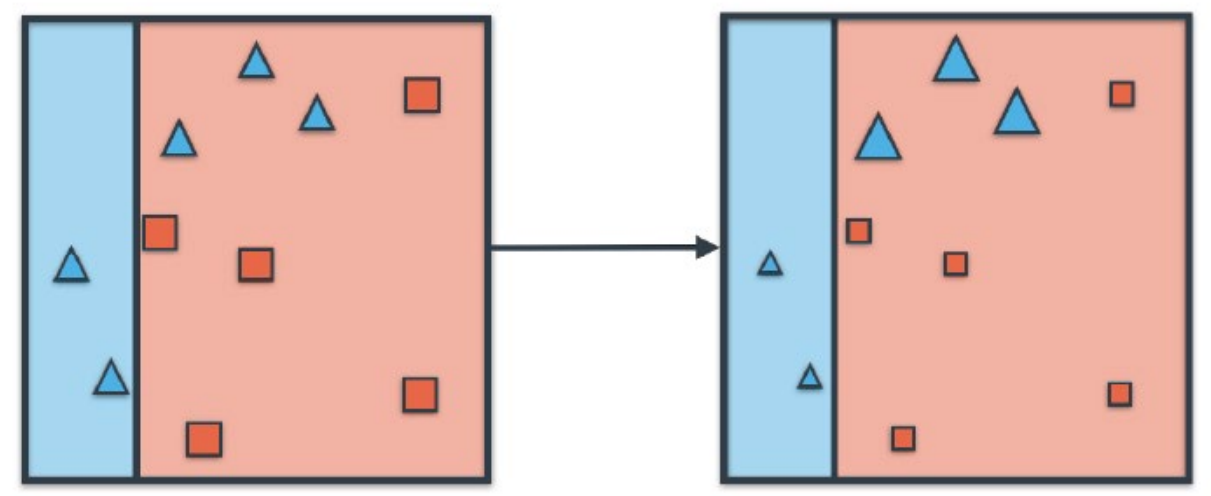

Figure 7: Shrinking weights of correctly classified points and enlarging misclassified points

This creates a dataset with weighted data points. Next, a second learner is fit on this weighted dataset. The next learner naturally tries to fit well on the misclassified data points of previous learner better. This process continues until a set of learners are generated each of which is better than the weakness of previous one.

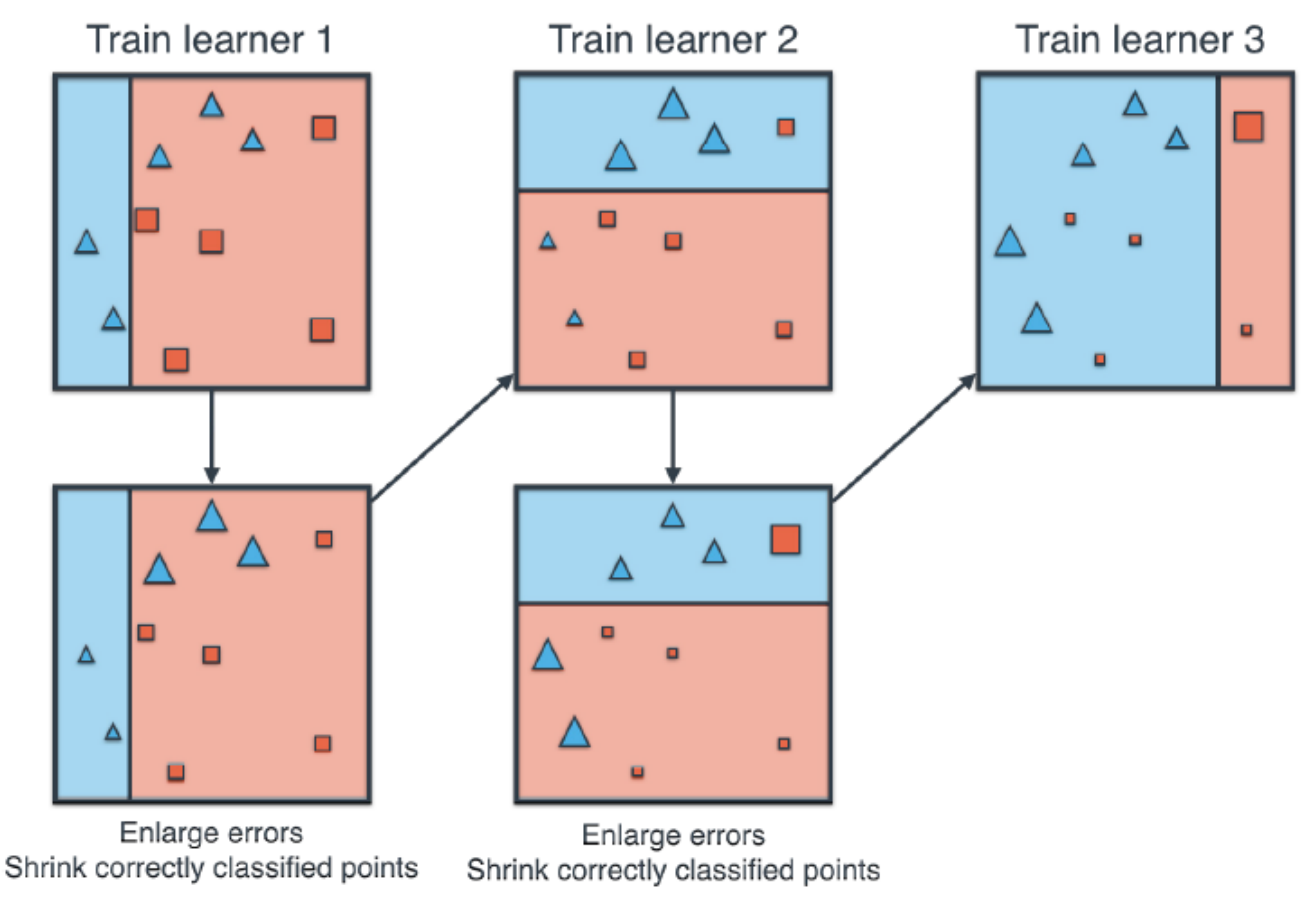

Figure 8: Subsequent weak learners of Adaboost learning on the mistakes of previous learners 
Finally, the classifiers vote during classification.

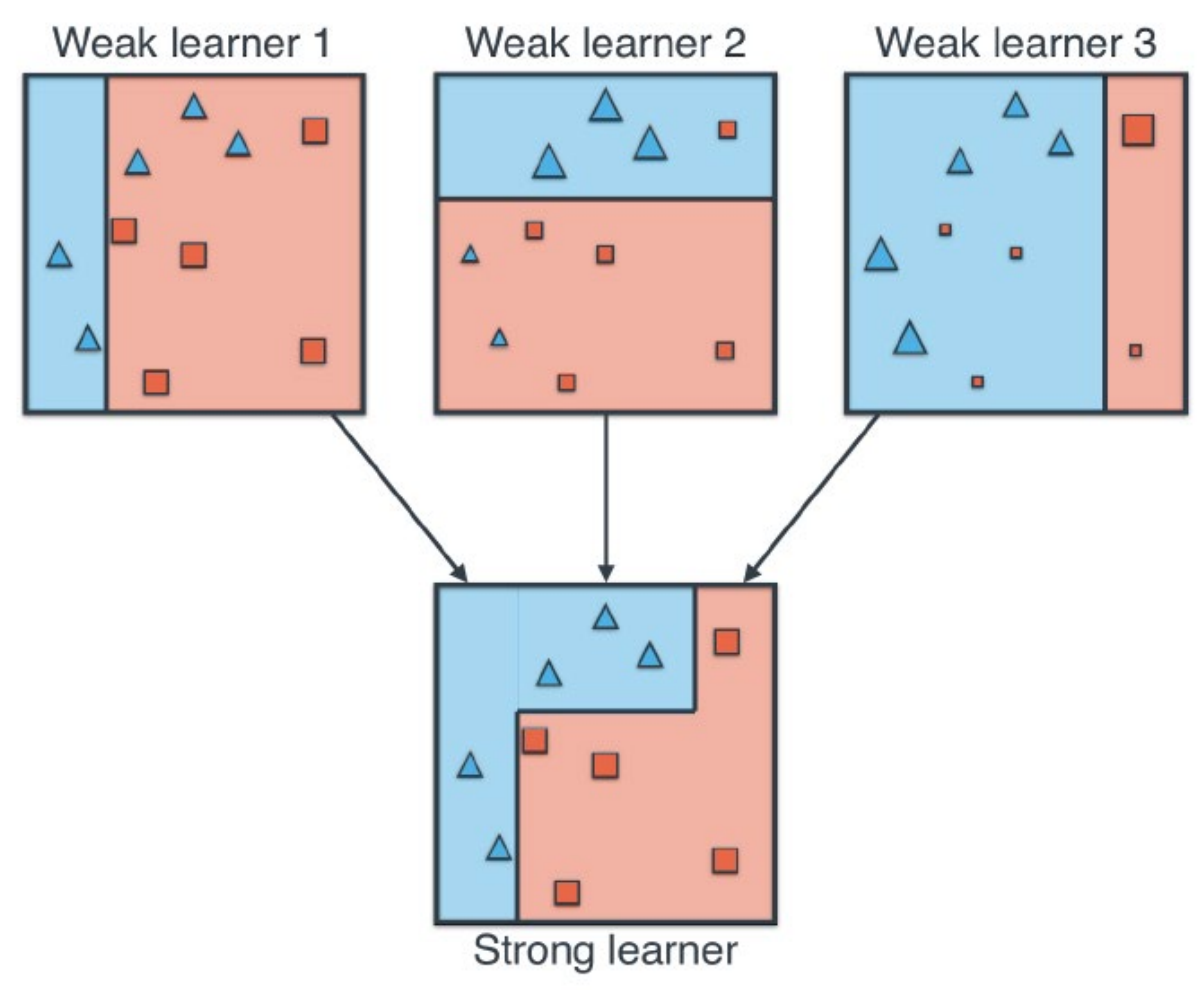

Figure 9: All weak learners finally vote to form a strong learner

AdaBoost is one of the most popular Boosting algorithms available right now. Developed by Freund and Shapire in 1997, AdaBoost turned out to be a very powerful boosting algorithm that has produced great results. In AdaBoost, all the weak learners are the simplest possible learner one can have: a decision tree of depth one, or a stump. A stump is usually represented by either a vertical or a horizontal line that splits data. It is the classifier that picks only one of the features, and classifies the data based on if that feature is smaller or larger than some threshold. 
Individual classifiers might not be capable of predicting the object class with precision, but a combination of many weak classifiers with each weak classifier learning from the mistakes of previous weak classifier, a strong model can be built. The base classifier to choose can be any standard classifier. Some of the most common standard classifiers include Decision Trees and Logistic Regression etc. A weak classifier is one which generates better results than a random guess but still is unable to classify the targets to their classes. AdaBoost, instead of being a model perse, can be used along with other classifiers to help learn from their mistakes and build a stronger better performing model. For all the reasons specified, AdaBoost is considered the best "out-of-box" classifier.

\section{i. An Example of How AdaBoost Works}

Step 1: Samples from the training data are weighted and a weak classifier like Decision Tree is fit onto this training data. Each sample's weight talks about the weightage of necessity in correctly classifying the sample. Firstly, for all the samples, equal weights are assigned.

Step 2: A decision stump is created for every existing variable and the stumps are observed to check how well they classify the data into their appropriate classes.

Step 3: For misclassified samples, their weightage is increased which forces the next stump to properly classify these misclassified points. Each weak classifier is also assigned a weight to indicate how well the classifier is performing. Classifiers with better performance are assigned higher weightages. 
Step 4: Keep repeating the process from step2 until a desired level of epochs has been reached or almost all the samples from the data have been appropriately classified.

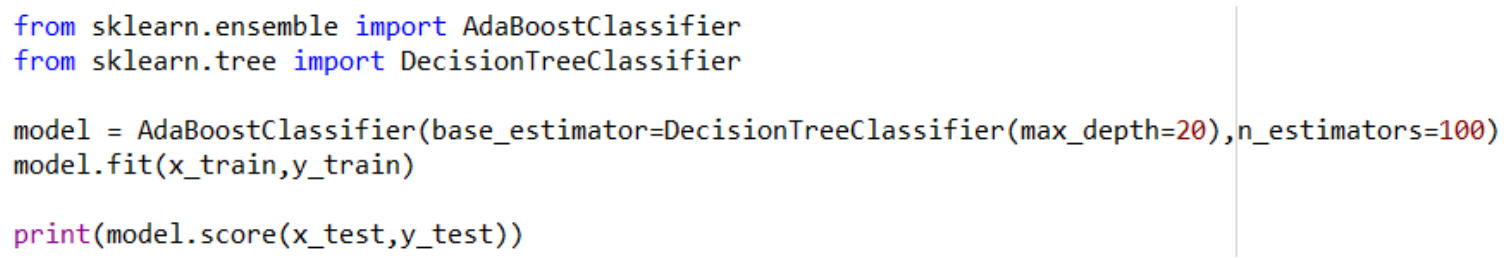

Figure 10: Training an Adaboost classifier on sklearn with base classifer as Decision tree

\section{B. SVM}

The objective of the support vector machine algorithm is to find a hyperplane in an Ndimensional space ( $\mathrm{N}$ - the number of features) that distinctly classifies the data points. SVM is a supervised machine learning model that is inherently a two-class classifier. However, as most problems involve multiple classes, a multiclass SVM is often used. A multiclass SVM forms multiple two class classifiers and differentiates the classifiers based on the distinct label vs. the rest (one-vs-rest or one-vs-all) or between each pair of classes (one-vs-one). SVM performs the classification by creating a hyperplane in such a way that separation between classes is as wide as possible. For SVM, the best hyperplane is the one that maximizes the margins from both tags. In other words, the hyperplane whose distance to the nearest element of each tag is the largest. 


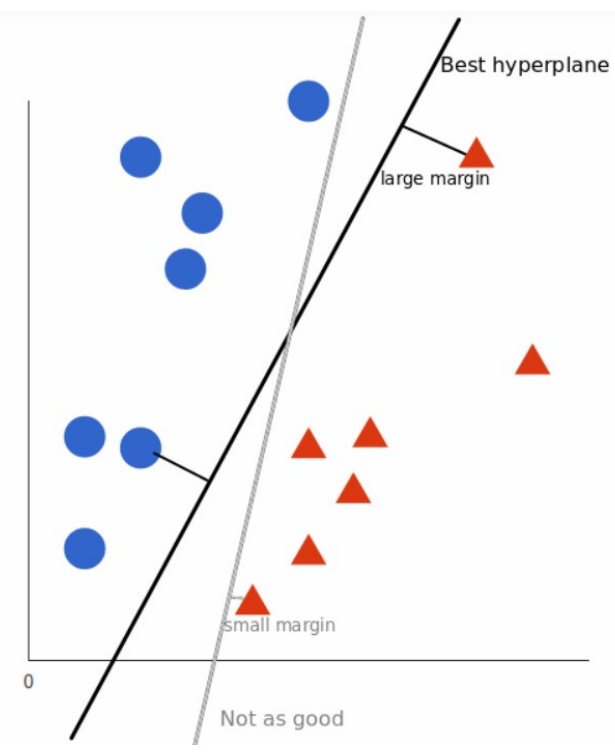

Figure 11: SVM trying to identify the best linear hyperplane that maximizes distance between classes

Sometimes, the datapoints do not observe a linear decision boundary but they might look clearly segregated and easily separable with a nonlinear hyperplane as shown in Fig 12.

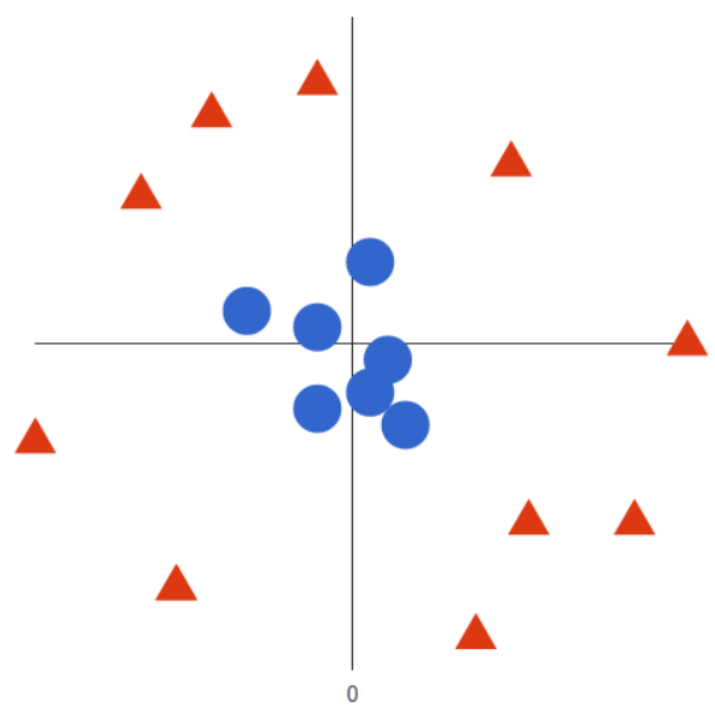

Figure 12: A complicated dataset impossible to be classified with a linear hyper plane. But blue and red points have clear distinction and separation between them. 
In such cases, SVM finds a clever way to classify nonlinear data by intelligently mapping the problem space into a higher dimension. This transformation is performed by a kernel function and is usually called the kernel trick. Since usually the kernel is linear, we always obtain a linear classifier. However, by using a nonlinear kernel a nonlinear classifier can be generated without transforming the data perse.

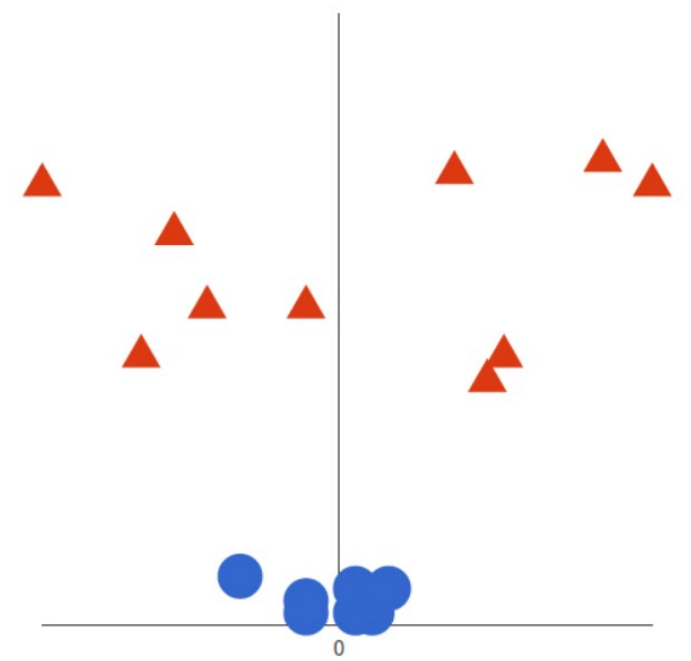

Figure 13: SVM projecting red datapoints to a higher dimension which facilitates easy classification.

As shown in fig 13, the problem domain is transformed into a higher dimension which makes it easy to identify a hyperplane that separates the two classes.

$$
\min _{w} \lambda\|w\|^{2}+\sum_{i=1}^{n}\left(1-y_{i}\left\langle x_{i}, w\right\rangle\right)_{+}
$$

Figure 14: Loss function that SVM tries to minimize between hyperplane and target points.

This loss function that SVM tries to minimize is called the square hinge loss function. 


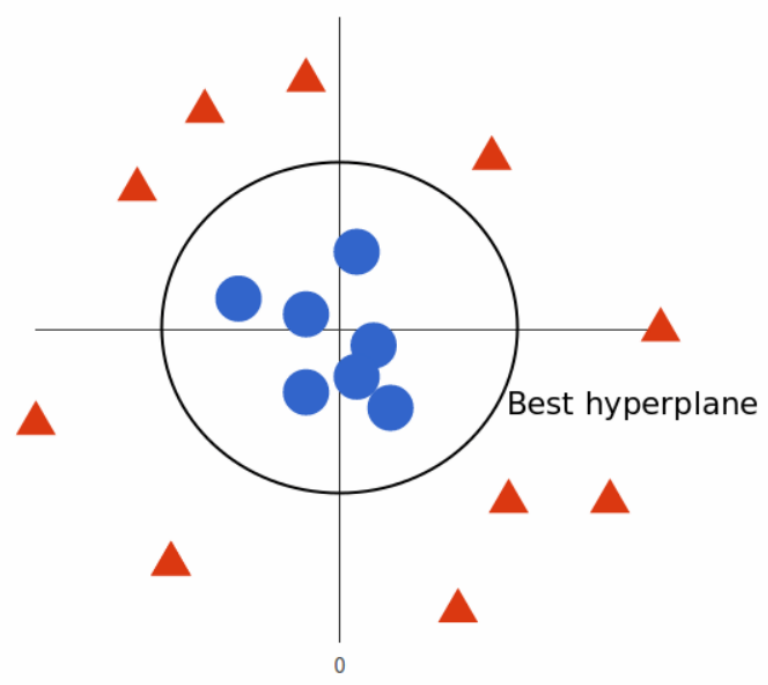

Figure 15: SVM identifying the best hyperplane after performing the kernel trick

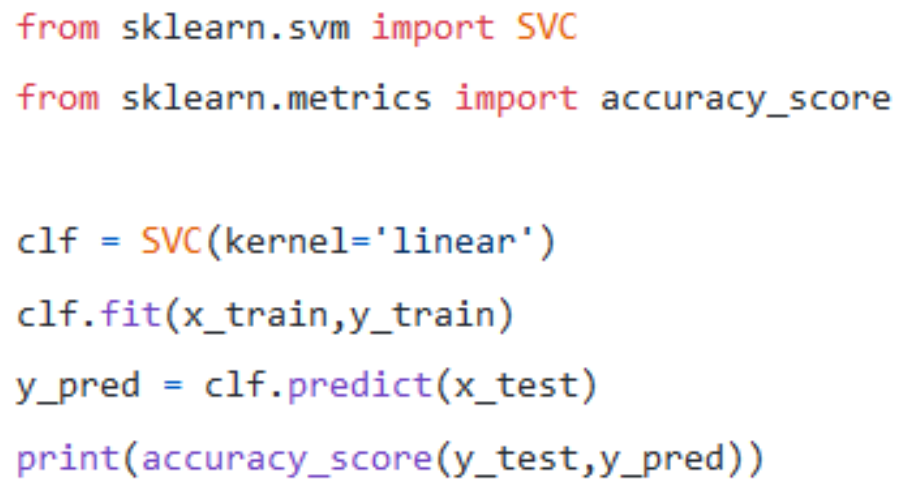

Figure 16: Implementing an SVM classifier with sklearn.

\section{ConvNet (CNN)}

CNN's are some of the most popular DL algorithms (Deep Learning) and have consistently won the ILSVRC challenges. CNN's feed on image input data. It trains and allocates weights to specific features of the image learnt through kernels or filters. Unlike other classification algorithms, CNN's do not require much effort in the pre-processing 
phase. Initially before the advent of CNN's, image features were hand-engineered, and this became too complicated. CNN's on the other hand, automatically learn these features during the training phase. CNN's architecture is similar to the arrangement of neurons in the visual cortex. Similar to how neurons that are wired together, fire together, the nearby pixel of an image forms a portion of a receptive field and have a semantic meaning. The spatial and temporal dependencies of an image can be captured with the help of filters or kernels. CNN's work better with image data since the trained weights can be reusable with transfer learning and also the number of parameters are greatly reduced compared to Multilayer Perceptrons. In a nutshell, CNN's can be trained to identify the features of an image better than other architectures.

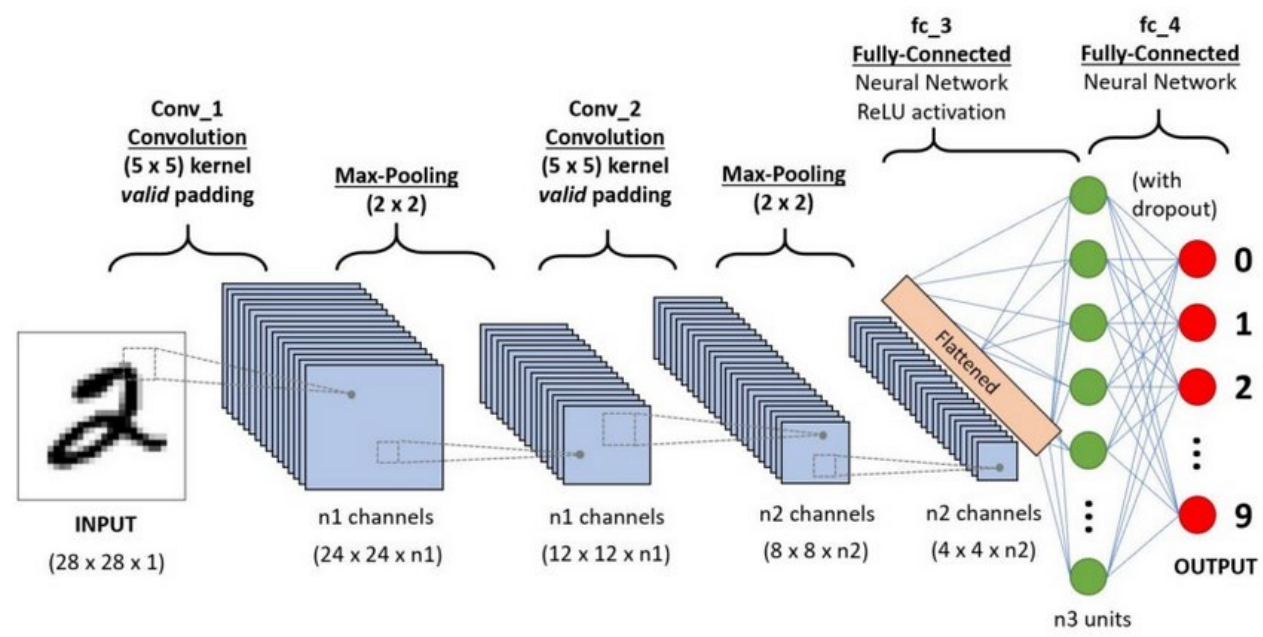

Figure 16: CNN classifying handwritten digits (Saha, S. (2018, December 17). A comprehensive guide to convolutional neural networks - the eli5 way. Retrieved April 28, 2021, from https://towardsdatascience.com/a-comprehensive-guide-to-convolutional-neural-networks-the-eli5way-3bd2b1164a53)

CNN's use filters to catch the importance of surrounding pixels. A filter is a matrix of numbers that capture certain characteristics from an image. A user specified filter size usually 3.3 or $5 \times 5$ is filled with random numbers and is scanned through the whole image 
from top left corner to the bottom right corner. At every point in the image, a convolutional operation is performed with the numbers in the filter.

Filters chosen can be used to represent anything. For example, for a picture of humans, one possible filter is the human eye and the values of the eye filter denote the strong presence of eye in the source image and also explains the number of times and the location of eye in the image. Due to this, the number of weights needed to be learnt reduces drastically compared to a Multi-Layer Perceptron. This facilitates the presence of feature anywhere in the image without confusing the neural network.

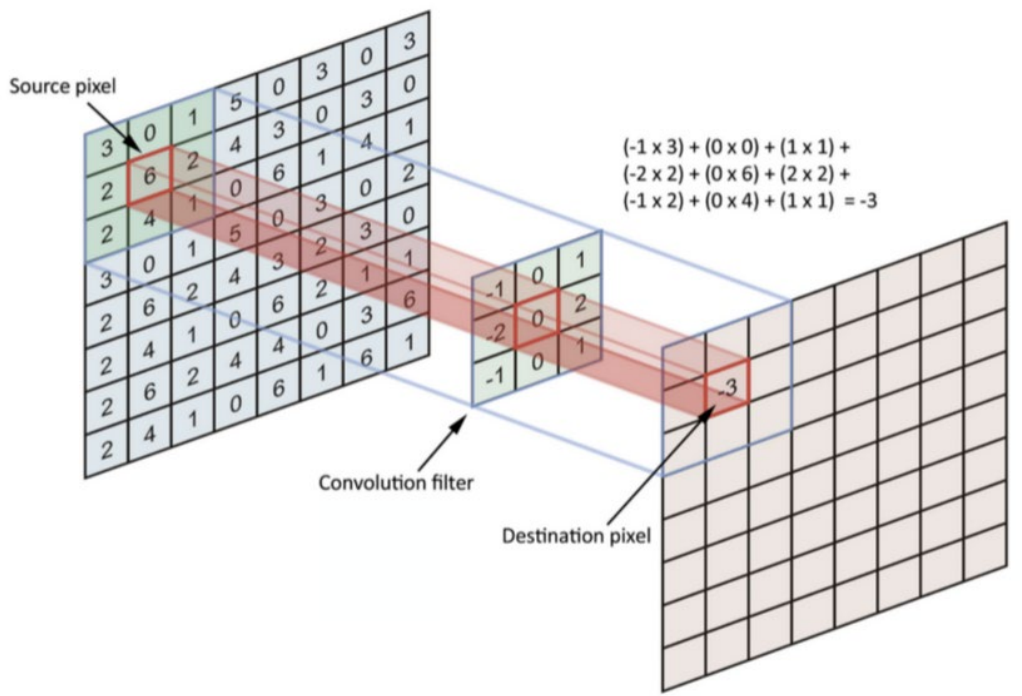

Figure 17: Convolution operation performed by a filter on the original image (Saha, S. (2018, December 17). A comprehensive guide to convolutional neural networks - the eli5 way. Retrieved April 28, 2021, from https://towardsdatascience.com/a-comprehensive-guide-to-convolutional-neuralnetworks-the-eli5-way-3bd2b1164a53)

After the processing of all filters on the source image, every filter generates a feature map. On these feature maps, an activation function is applied which indicates the presence or absence of a certain feature in the image. For a deeper Convolutional 
Neural Network, more filters and more feature maps can be added which become more and more abstract with the depth of the network. Max pooling and average pooling layers are the pooling layers following the convolutional layers. They help in selecting the largest or average values of the feature maps and these values are fed to the succeeding layers. Although any operation can be performed with pooling layers, max pool is the most common operation which helps in finding outliers.

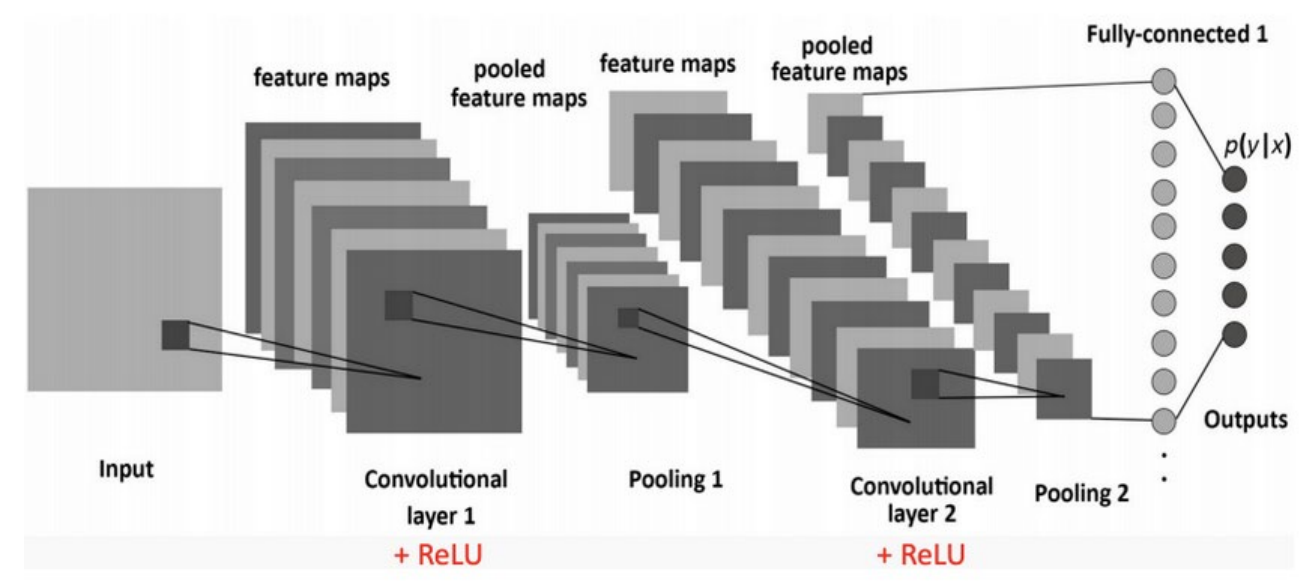

Figure 18: All different layers in a convolutional neural network. (Saha, S. (2018, December 17). A comprehensive guide to convolutional neural networks - the eli5 way. Retrieved April 28, 2021, from https://towardsdatascience.com/a-comprehensive-guide-to-convolutional-neural-networks-the-eli5way-3bd2b1164a53)

The basic idea of a $\mathrm{CNN}$ is to intelligently adapt to the properties and features of an image:

- Surrounding pixels are more important and hide semantic meanings.

- Points of interest can be located at any part of the image.

CNN's are also composed of layers, but those layers are not fully connected: they have filters, sets of cube-shaped weights that are applied throughout the image. Each 2D slice 
of the filters are called kernels. These filters introduce translation invariance and parameter sharing and these filters are applied through convolution operations.

\section{1) Convolutional Layer}

A convolution layer is where a kernel is applied on the input with a certain stride to obtain the convolved feature (see Figure $\mathrm{x}$ ). A kernel is essentially a set of weights initialized randomly and tweaked by the network during backpropagation based on the loss function. When the kernel is applied on the input, a dot product is performed between the kernel weights and the region it is being applied on. Therefore, the convolved feature is the sliding dot product of the input image from top to bottom as shown in Figure 9.

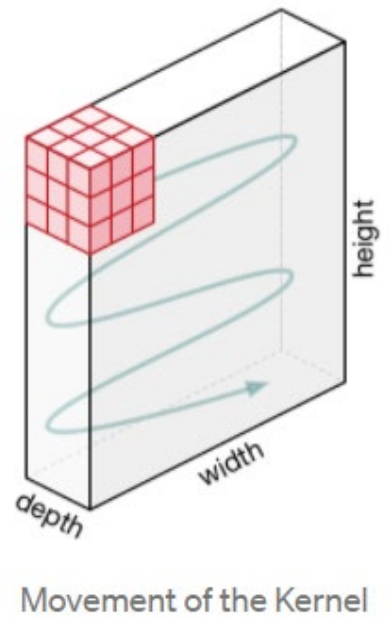

Figure 19: Movement of kernel performing convolutions across the original image. (Saha, S. (2018, December 17). A comprehensive guide to convolutional neural networks - the eli5 way. Retrieved April 28, 2021, from https://towardsdatascience.com/a-comprehensive-guide-to-convolutional-neural-networks-theeli5-way-3bd2b1164a53)

The kernel has the same depth as the input it is being applied on. For a grayscale image, the depth of the kernel is 1 whereas for a color image with three channels (RGB - Red, Green, and Blue), the depth of the kernel would be 3. Therefore, three kernels would be 
applied to three different channels. Figure 20 shows how three different kernels with different set of weights are being applied on the three channels of the image. The dot product of all the three channels is summed up with bias to obtain the feature output.
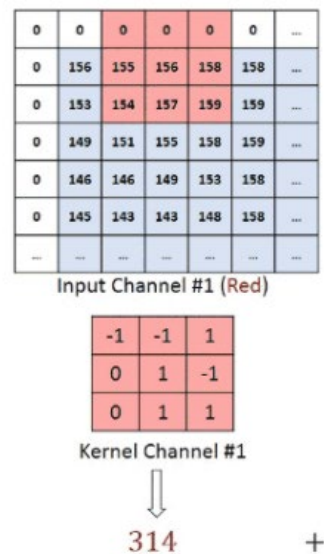
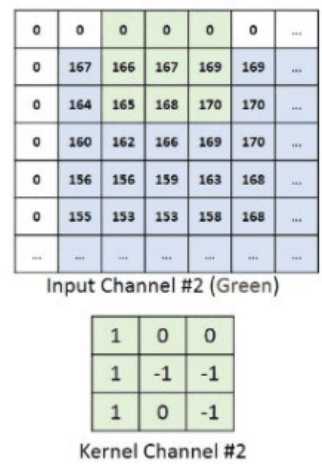

Kernel Channel \#2$$
-175
$$

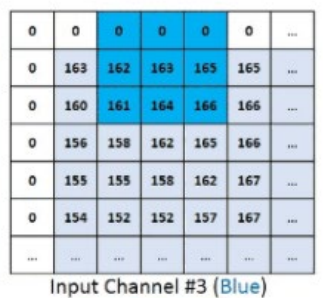

Input Channel \#3 (Blue)

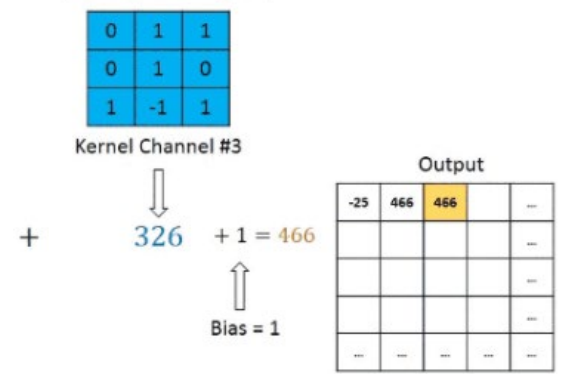

Convolution operation on a MxNx3 image matrix with a $3 \times 3 \times 3$ Kernel

Figure 20: Convolution operation on a MxNx3 image matrix with a 3x3x3 kernel (Saha, S. (2018, December 17). A comprehensive guide to convolutional neural networks - the eli5 way. Retrieved April 28, 2021, from https://towardsdatascience.com/a-comprehensive-quide-to-convolutional-neural-networks-the-eli5-way3bd2b1164a53)

There can be multiple Convolutional layers in a CNN. The initial Convolutional layers extract the low-level features of an image such as edge, color, and gradient orientation. The deeper Convolutional layers extract more complex high-level information such as shapes, objects etc. When a convolution is performed, the type of padding is also chosen. There are two types of padding called as same padding and valid padding. Same padding does not reduce the dimensionality of the input and is performed by padding all sides of the image with zeroes. On the other hand, to reduce the dimensionality of the input, "valid padding" can be selected. 


\section{2) Pooling Layer}

A Pooling layer is applied after the Convolutional layer to reduce the dimensionality and capture the most relevant information. There are two types of pooling that are commonly used: Max Pooling and Average pooling. Max pooling only selects the maximum value of the region on which the kernel is applied. Average pooling computes the average of all the values of the region on which the kernel is applied. Max pooling is heavily used as it also eliminates noise along with reducing the dimensions as we discard the less useful information. Figure $\mathrm{x}$ shows the result of a $2 \times 2$ max pooling and a $2 \times 2$ average pooling with a stride of 2 being applied an input feature map.

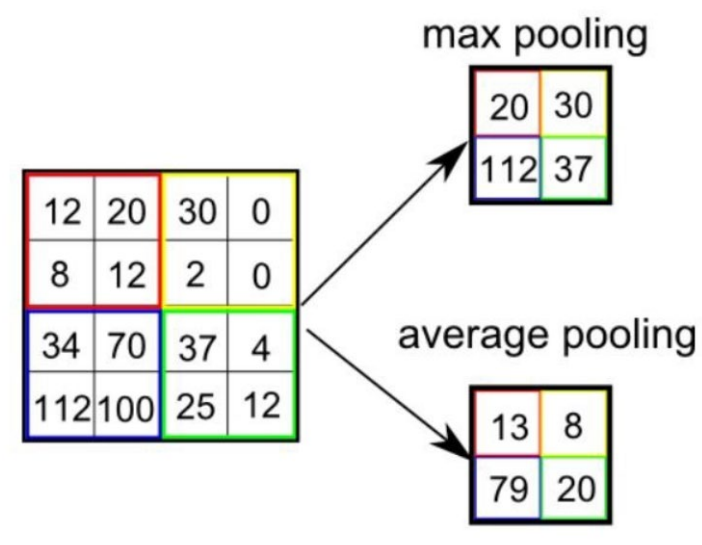

Types of Pooling

Figure 21: Max pooling and average pooling on input feature map (Saha, S. (2018, December 17). A comprehensive guide to convolutional neural networks - the eli5 way. Retrieved April 28, 2021, from https://towardsdatascience.com/a-comprehensive-quide-to-convolutional-neural-networks-the-eli5-way3bd2b1164a53)

\section{3) Fully Connected Layer}

Fully connected layers are used after the convolutional layers to capture the non-linear properties of the features obtained by the convolutional layers. The output is flattened 
before feeding it to the Fully connected layers. The fully connected layers are typically followed by a SoftMax layer to perform classification.

Different variations of Convolutional Neural Networks have been developed overtime with the intent to reduce training time, improve accuracy, and deal with the vanishing gradient problem that is common in Deep Convolutional Neural Networks. One of the earliest and most popular CNN models is the VGG-16 which we used in our project.

\section{A. $V G G-16$}

VGG-16 was the winner of the ImageNet competition in 2014. It uses a 3x3 filter with a stride of 1 and padding as "same". It uses a $2 \times 2$ filter for max pooling with a stride of 2 . It uses 13 convolutional layers followed by 2 fully connected layers and a softmax layer (see Figure 12). It has about 138 million parameters due to which the training time is much higher compared to other CNNs.

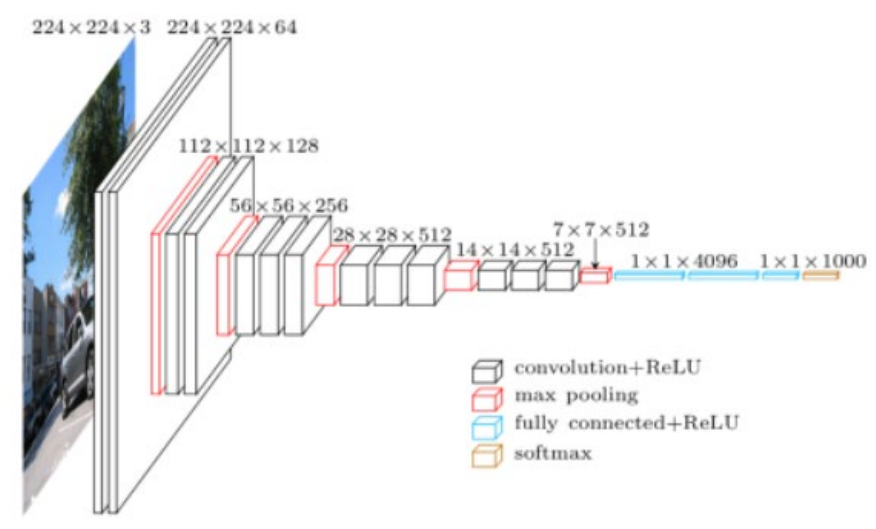

Architecture of VGG16

Figure 22: VGG 16 Architecture (Thakur, R. (2020, November 24). Step by step VGG16 implementation in Keras for beginners. Medium. https://towardsdatascience.com/step-by-step-vgg16implementation-in-keras-for-beginners 
K. Simonyan and A. Zisserman developed VGG16, a convolutional neural network which was one of the most popular models submitted in the ILSVRC challenge 2014. They talked about the model in a paper titled "Very Deep Convolutional Networks for LargeScale Image Recognition" from the University of Oxford. VGG16 reached a top accuracy of $92.7 \%$ for a dataset named ImageNet, which contains around 14 million images belonging to 1000 classes. It improved over Alex Net by changing the kernel filters of size 11 and 5 in the first and second layers of the neural network with multiple kernel filters of size 3x3. VGG16 used NVIDIA Titan Black GPUs to train for weeks. The first convolutional layer of VGG16 is fed an RGB image of fixed size 224x224. The RGB image is sent through a set of convolutional layers, with small receptive field filters of size $3 \times 3$. In one of the configurations, it makes use of a convolutional filter of size $1 \mathrm{x} 1$ which performs a linear transformation and then a non-linear transformation. A convolutional stride of 1 pixel is fixed and the spatial resolution is conserved after convolution, i.e., for convolutional layers of size $3 \times 3$ padding is set to 1 pixel. Max pooling layers follow some of the convolutional layers and the five max pooling layers help in carrying out spatial pooling. The max pooling operation happens over a pixel window of size $2 \times 2$ and the operation is carried out with stride 2 .

In VGG16, three exists three FC layers. These layers contain neurons that are fully connected and are followed by a sequence of convolutional layers. The two initial convolutional layers contain 4096 neurons each, and since the third FC layer performs the 1000 class ImageNet classification, it thus contains 1000 channels. The FC layers are followed by the SoftMax layer which happens to be the final last layer in the network. In 
all the networks, the configuration parameters of all fully connected layers remain the same. The hidden layers contain a ReLU non-linear transformation activation function which transform linearity into non-linearity. 


\section{EXPERIMENTAL METHODS}

In the task classification, we designed our study to classify four tasks from the fixation and free-viewing datasets.

\section{A. Experiment 1}

\section{i. AdaBoost Model}

We replicated Kumar et al. model in [6] using the same architecture described in their study. However, we included the free-viewing dataset as well to test the accuracy of the classification model they suggested on a different task that includes a visual search task (Waldo, Puzzle) or a free visual exploring (Natural Scene). We followed Kumar and his colleague in labeling each task as (0-waldo, 1-puzzle, 2-blank, 3-natural) for the 4 tasks in both conditions. The model used each timestamp's five variables (LXpix, LYpix, LXhref, LYhref, LP) and a label as a single exemplar. The accuracy result for the fixation condition corroborates Kumar et al results of $95 \%$. The accuracy for classifying the four tasks for the free-viewing condition is $84 \%$.

\section{ii. Support Vector Machine (SVM) Model}

In this model, we decided to use the RGB images we generated from the eye fixations. SVM's are powerful classifiers that create hyperplanes to classify the data into different classes. The kernel used in SVM makes this model a practical and powerful model for solving linear and non-linear problems. We fed the images directly to the SVM model. In all the images, the features are represented by the pixels themselves. The 224 by 224 sized image is reshaped into a 1x50176 vector as shown in Figure 2. Then the vector was input into the SVM. This method has an accuracy of $74 \%$ for both conditions. 

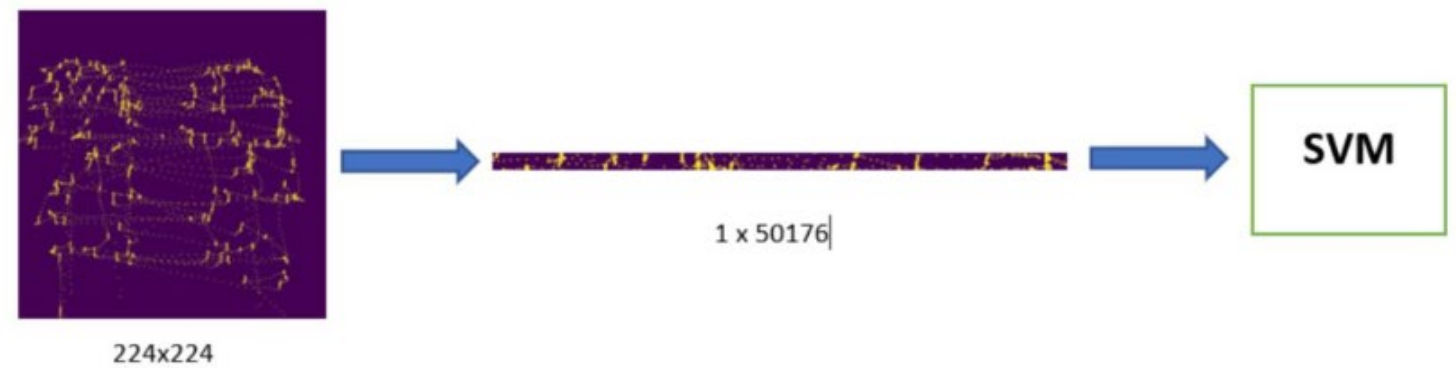

Figure 23: Support Vector Machine using RGB images of scan path.

\section{iii. ConvNet + SVM Model}

We used ConvNet known as VGG16 with SVM to be able to extract the important features from the images. ConvNet transforms one volume of activations to another through a differentiable function. In the method described in 4.1.2, we used the original images. In this model, we extracted the most important features of each image using convolutional layers. Then, we fed those features to the SVM. A technique that is known as bottleneck feature extraction. Bottleneck features are the last activation maps before the fully connected layers as shown in figure 24. Each convolutional layer learns the representations of the image. At the end of the convolutional layers, these learned feature maps were flattened into a vector at the second dense layer. The flattened vector from the second dense layer was fed into the third and final dense layer for the classification model. The third dense layer (SoftMax layer) classified the data based on these features. 


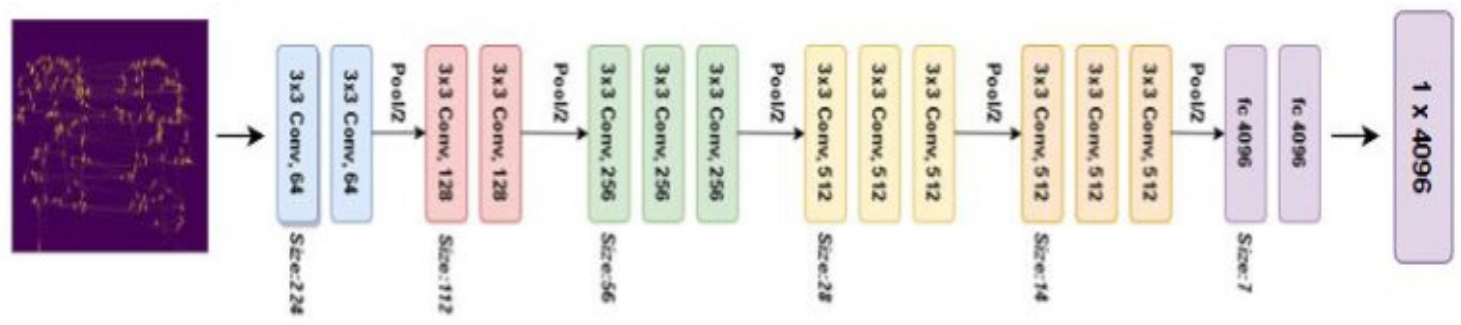

Figure 24: The proposed ConvNet (VGG-16) architecture. The number of convolutional filters and neural network connections for each layer are as shown.

Therefore, $224 \times 224$ images, in a nutshell, can run through the network. Then, the image converted into a 1x4096 vector. Each vector is fed into a classification layer. The vector at the end of the second dense layer contained important information captured by the previous convolutional layers. For every original image, we run it through the VGG16 network and capture its features at the output of the second dense layer. The model was trained with 4fold cross-validation (75:25 training set: test set) and the accuracy of the four models were averaged. Figure 4 shows the features fed as inputs to the SVM. The resulted accuracies were $36.6 \%$ and $88 \%$ for the fixation condition and free-viewing condition, respectively.

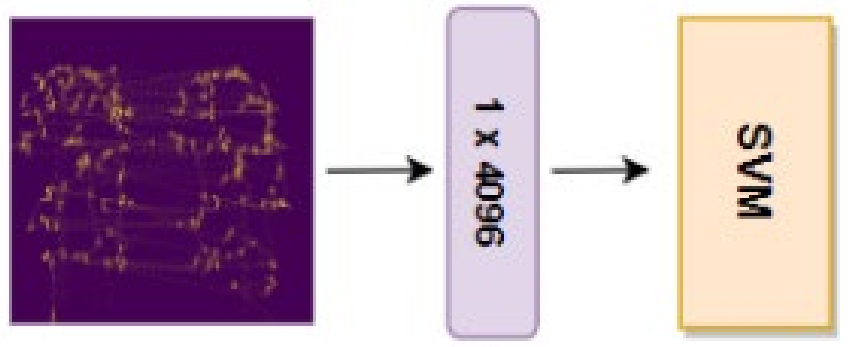

Figure 25: SVM model taking input bottleneck features from the last layer of VGG-16 CNN model. 


\section{B. Experiment 2}

In this experiment, we used the same three models described in Experiment 1. We removed only the natural scene task from the dataset as we want to test how deep learning and machine learning-based models would classify tasks that involve visual search (Waldo, and Puzzle), or blank task. The goal of this experiment is to determine if the three models misclassified between scan paths that represent visual search and scan paths that represent visual exploration in Experiment 1. We ran every model using the three tasks (Waldo, Puzzle, Blank) on the free-viewing condition and fixation condition. The accuracies achieved by SVM, AdaBoost, and ConvNet+SVM were $80 \%, 97 \%, 40.55 \%$ for the fixation dataset, and $76 \%, 88 \%, 97 \%$, for the free-viewing dataset, respectively.

\section{Experiment 3}

In this experiment, we included only tasks that require visual search and removed the Blank and Natural Scene tasks from both conditions. We ran the three models described in Experiment 1. Table 1 summarized the accuracies obtained from Experiment 1 and Experiment 2, and Experiment 3. 
Table 3: Final accuracies of all models

\begin{tabular}{|c|c|c|c|c|}
\hline Experiment & Tasks & Model & $\begin{array}{l}\text { Accuracy } \\
\text { (Free- } \\
\text { Viewing } \\
\text { Condition) }\end{array}$ & $\begin{array}{l}\text { Accuracy } \\
\text { (Fixation } \\
\text { Condition) }\end{array}$ \\
\hline \multirow[t]{3}{*}{1} & 4 & SVM & 74 & 74 \\
\hline & 4 & AdaBoost & 84 & 95 \\
\hline & 4 & ConvNet + SVM & 88 & 36.6 \\
\hline \multirow[t]{3}{*}{2} & $\begin{array}{l}3 \text { (Waldo, Puzzle, } \\
\text { Blank) }\end{array}$ & SVM & 76 & 80 \\
\hline & 3 & AdaBoost & 88 & 97 \\
\hline & 3 & ConvNet + SVM & 97 & 40.55 \\
\hline 3 & 2 (Waldo, Puzzle) & SVM & 88.75 & 24.6 \\
\hline 3 & 2 & Ada Boost & 89.3 & 98 \\
\hline 3 & 2 & ConvNet + SVM & 98.33 & 60 \\
\hline
\end{tabular}

\section{Discussion}

In Experiment 1, the AdaBoost model was successful in classifying tasks in the fixation condition but was not efficient in classifying tasks that require a visual search. The filtered eye movement data used by AdaBoost has pupil size as one of the features. When a user 
only fixates at the center of the screen, the pupil size could be affected by the overall brightness or the luminance of the stimulus more than visual search or cognitive load level [15]. Therefore, the classification using the eye movement data will depend mostly on the pupil dilation, given the fact that the $\mathrm{x}$ and $\mathrm{y}$ positions of the fixations will not vary across the tasks in the fixation condition (see Figure 1). Another possibility that the users could try to do a minimal search during the Puzzle or Waldo tasks in the fixation condition while maintaining to look at the center. That creates a small scan path around the center of the screen [17], [4]. The confusion matrix on Figure 5 (A) for the free-viewing condition shows that misclassification of a task is associated more with the natural scene task for ConvNet in Experiment 1. When we included the natural scene images in the dataset for the SVM and ConvNet+SVM models, either the natural scenes task was predicted as other tasks, or the other tasks were predicted as the natural scene task. This result shows that the scan path images generated for the natural scene task cannot be uniquely identified.

The natural scene task does not have a target like the Waldo task, nor multiple targets like the Puzzle task, so the scan path that resulted from the exploration of the scene was difficult to be classified by ConvNet. During the natural scene, the ConvNet was not able to differentiate between the scan paths from eye fixations concentrated on salient parts of the image (Natural scene), the Large predominant horizontal saccades (Puzzle), or the concentration of the fixation around the target (Waldo) [19]. In Experiment 2, the ConvNet+SVM model showed a significant improvement and the highest accuracy when the natural scene was removed from the free-viewing dataset (see Figure 5 (B)), while the other two models did not improve at the same level as the ConvNet. This provides evidence 
that deep learning can classify tasks that require visual attention levels more efficiently than other machine learning-based methods. For the fixation dataset, AdaBoost and SVM show that when the visual task is not complex, then deep learning would not be a robust tool.

Experiment 3 showed that eye movement data collected from visual search tasks can be classified by the deep learning model (see Figure $5(\mathrm{C})$ ). This corroborates what Yarbus suggested about complex mental states being inferable from scan paths [1]. We show that non-complex tasks are classified with higher accuracy when using machine learning algorithms such as AdaBoost.

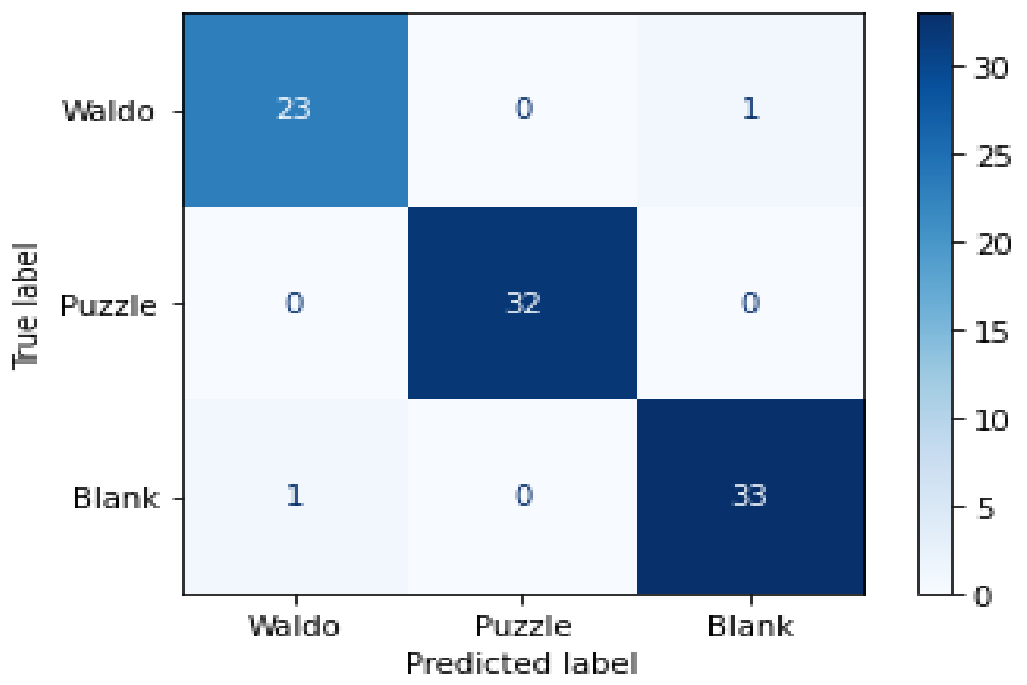

Figure 26: Confusion Matrix of ConvNet for the free-viewing dataset for 4 tasks, 


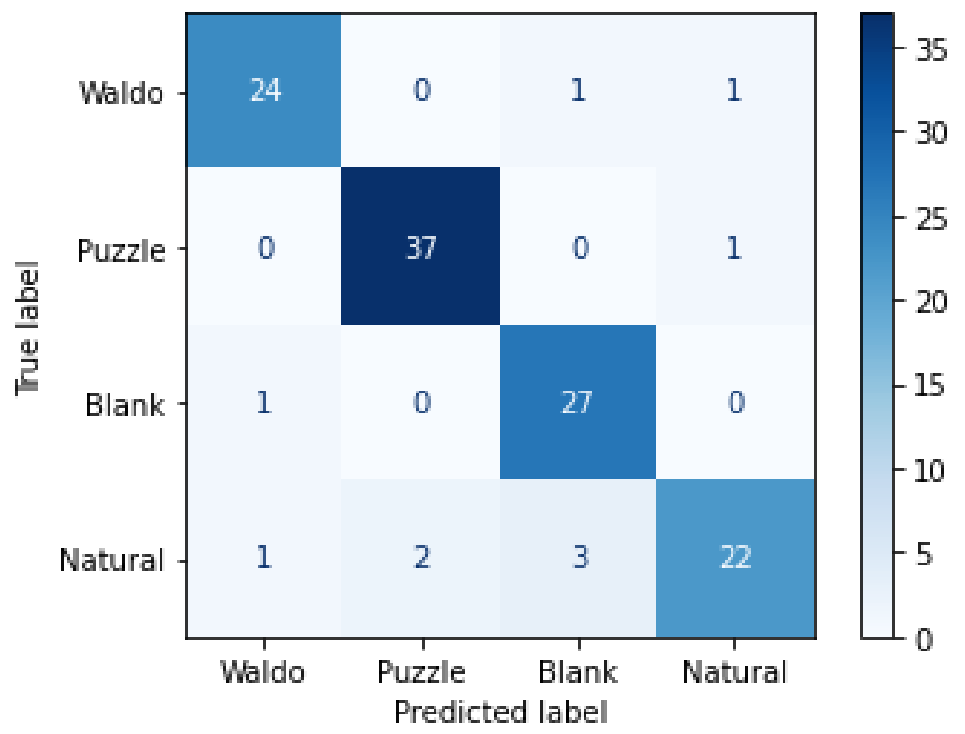

Figure 27: Confusion Matrix of ConvNet for the free-viewing dataset for 3 tasks,

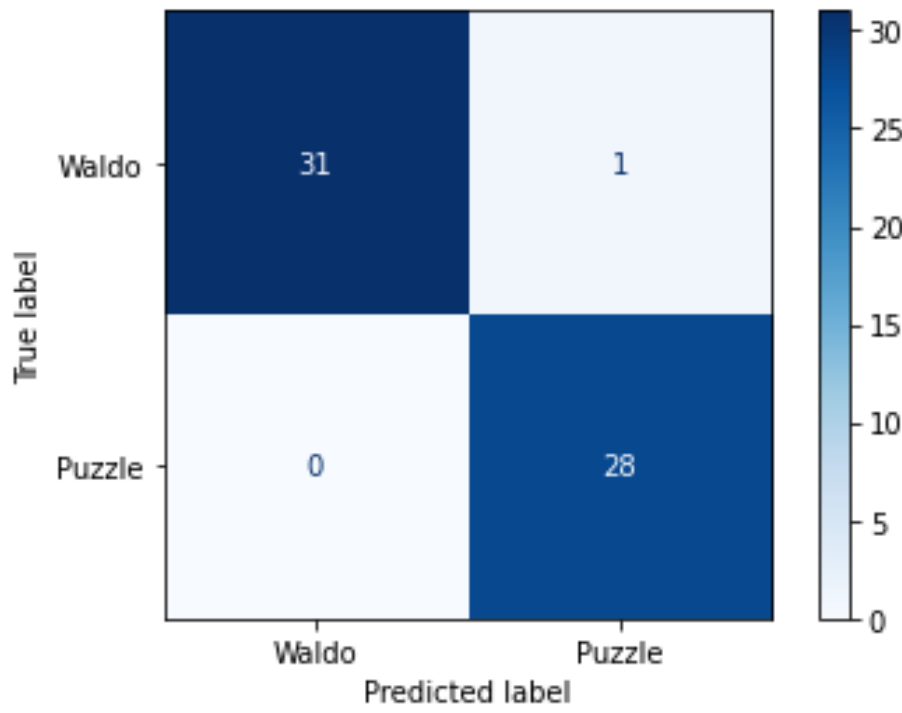

Figure 28: Confusion Matrix of ConvNet for the free-viewing dataset for 2 tasks.

The same process however did not yield good results for fixation data as the RGB mapped images generated for fixation data looked similar (see Figure 29) across all 4 scenes of Natural, Blank, Waldo and Puzzle. This is due to the reason that during fixation condition, 
subjects usually have a fixation point to look at and hence the gaze points are concentrated at the center. Since all the images are similar, it becomes impossible to employ image classification algorithms.
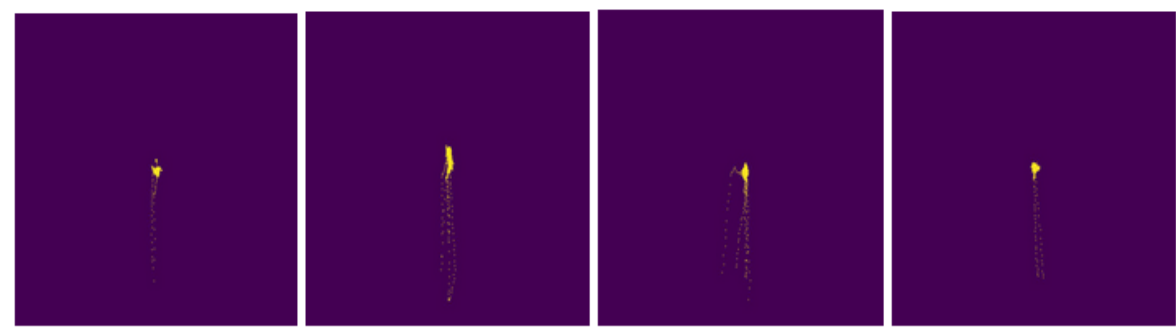

Figure 29: RGB images for fixation data for Waldo, Natural, Puzzle and Blank scenes. 


\section{CONCLUSION AND FUTURE WORK}

In this study, we use three different classification models to classify four tasks. Each of the four tasks was performed in two conditions where subjects either look only in a fixation mark at the center of the screen or do a visual search or visual exploration. Our experiments show that the deep learning model works best to classify visual search tasks. However, the deep learning model was impaired when the visual exploration task was a part of the dataset. Machine learning-based methods work well with tasks that require minimal visual attention level tasks. This study provides evidence that models for task classification should be selected based on the type of visual attention that the user devotes to the tasks, and one model cannot be the best approach to classify tasks of different visual attention levels. Future work should explore a wide variety of tasks such as visual exploration, reading, etc. which entail varying visual attention levels. The ability to classify visual tasks from eye movement data with high accuracy can help to improve intelligent user interfaces such as gaze-supported level and media retrieval [8], user interaction using mobile phones [10], cognitive assistance for patients [7], to name a few. 


\section{REFERENCES}

[1] Alfred L Yarbus 2013, Eye movements and vision. Springer.

[2] Ali Borji, Laurent Itti. 2014. Defending Yarbus: eye movements reveal observers' task. Journal of Vision, 14(3):29.

[3] B Ali Borji, Laurent Itti. 2013. State-of-the-art in modeling visual attention. IEEE Trans. Pattern Analysis and Machine Intelligence (PAMI), 35, 185-207

[4] Brandt S. A. Stark L. W. 1997. Spontaneous eye movements during visual imagery reflect the content of the visual scene. Journal of Cognitive Neuroscience

[5] Anne M. Treisman. 1980. A feature integration theory of attention. Cognitive Psychology, 12:97-136.

[6] C. Ayush Kumar, Anjul Tyagi, Michael Burch, Daniel Weiskopf, and Klaus Mueller. 2019. Task classification model for visual fixation, exploration, and search. In Proceedings of the 11th ACM Symposium on Eye Tracking Research \& Applications (ETRA '19). Association for Computing Machinery, NewYork, NY, USA, Article 65, 1-4.

[7] Daniel Sonntag. 2015 "Kognit: Intelligent Cognitive Enhancement Technology by Cognitive Models and Mixed Reality for Dementia Patients." AAAI Fall Symposia.

[8] Hosnieh Sattar, Sabine Müller, Mario Fritz, Andreas Bulling, "Prediction of search targets from fixations in open-world settings," in 2015 IEEE Conference on Computer Vision and Pattern Recognition (CVPR), Boston, MA, USA, 2015 pp. 981-990.

[9] Jeremy M. Wolfe. 1994. Visual Search in Continuous, Naturalistic Stimuli.” Vision Research, 34(9):1187-1195,

[10] Julian Steil, Philipp Müller, Yusuke Sugano, Andreas Bulling. 2018. "forecasting User Attention During Everyday Mobile Interactions Using Device-Integrated and Wearable Sensors". Proc. ACM International Conference on Human-Computer Interaction with Mobile Devices and Services (MobileHCI), pp. 1:1-1:13 
[11] John M. Henderson, * Svetlana V. Shinkareva, Jing Wang, Steven G. Luke, and Jenn Olejarczyk (2013) Predicting Cognitive State from Eye Movements. PLOS ONE 8(5): e64937.

[12] Kyle R. Cave and Jeremy M. Wolfe. "Modeling the role of parallel processing in visual search.” Cognitive Psychology, 22:225-271, 1990..

[13] Michelle R. Greene, Tommy Liu, Jeremy M Wolfe. Reconsidering Yarbus: a failure to predict observers' task from eye movement patterns. Vision Res. 2012 Jun 1;62:1-8. doi: 10.1016/j.visres.2012.03.019. Epub 2012 Apr 2. PMID: 22487718; PMCID: PMC3526937.

[14] Michael B. McCamy, Jorge Otero-Millan, Leandro Luigi Di Stasi, Stephen L. Macknik and Susana Martinez-Conde. Highly informative natural scene regions increase microsaccade production during visual scanning. J Neurosci. 2014 Feb 19;34(8):2956-66. doi: 10.1523/JNEUROSCI.4448-13.2014. PMID: 24553936; PMCID: PMC6608512.

[15] Nada Attar, Matthew H. Schneps, and Marc Pomplun. "Working memory load predicts visual search efficiency: Evidence from a novel pupillary response paradigm.” Memory \& Cognition, 44(7):1038-1049, 2016.

[16] Nada Attar, Paul Fomenky, Wei Ding, and Marc Pomplun. "Improving Cognitive Load Level Measurement through Preprocessing of Psychophysical Data by Random Subspace Time-Series Method." In The IEEE International Conference in Human Computer Interaction

(ICHCI 2016), 2016.

[17] Nada Attar, Matthew H. Schneps, and Marc Pomplun Pupil size as a measure of working memory load during a complex visual search task Journal of Vision 13 (9), 160-160 (2), 2013

[18] O'Connell T. Walther D. (2012). Fixation patterns predict scene category. Journal of Vision, 12 (9): 801

[19] Otero-Millan J, Troncoso XG, Macknik SL, Serrano-Pedraza I, MartinezConde S. Saccades and microsaccades during visual fixation, exploration, and search: foundations for a common saccadic generator. J Vis. 2008 Dec 18;8(14):21.1-18. doi: 10.1167/8.14.21. PMID: 19146322.

[20] Shamsi T. Iqbal, Xianjun Sam Zheng, and Brian P. Bailey. 2004. Task-evoked pupillary response to mental workload in human-computer interaction. In CHI '04 Extended Abstracts on Human Factors in Computing Systems (CHI EA '04). Association for Computing Machinery, New York, NY, USA, 1477- 
1480.

[21] Yao Zhou, Jiamin Ren, Jingyu Li, Litong Feng, Shi Qiu, and Ping Luo. 2017. Video Classification via Relational Feature Encoding Networks. In Proceedings of the Workshop on Large-Scale Video Classification Challenge (LSVC '17). Association for Computing Machinery, New York, NY, USA, 913.

[22] Zelinsky G. Peng Y. Samaras D. (2013). Eye can read your mind: Decoding gaze fixations to reveal categorical search targets. Journal of Vision, 13 (14): $10,1-13$,

[23] Zhang L. Tong M. H. Marks T. K. Shan H. Cottrell G. W. (2008). Sun: A Bayesian framework for saliency using natural statistics. Journal of Vision, 8 (7): $32,1-20$,

[24] Zhao Q. Koch C. (2012). Learning visual saliency by combining feature maps in a nonlinear manner using adaboost. Journal of Vision, 12 (6): 22, 1-15, 
